\title{
A discrete strong discontinuity approach
}

\author{
D. Dias-da-Costa ${ }^{\mathrm{a}, *}$, J. Alfaiate ${ }^{\mathrm{b}}$, L.J. Sluys ${ }^{\mathrm{c}}$, E. Júlio ${ }^{\mathrm{a}}$ \\ a ISISE, Civil Eng. Dept., University of Coimbra, Rua Luís Reis Santos, 3030-788 Coimbra, Portugal \\ ${ }^{\mathrm{b}}$ IST and ICIST, Civil Eng. Dept., Instituto Superior Técnico, Av. Rovisco Pais, 1049-001 Lisboa, Portugal \\ ${ }^{\mathrm{c}}$ Delft University of Technology, Dept. of Civil Engineering and Geosciences, P.O. Box 5048, 2600 GA Delft, The Netherlands
}

\section{A R T I C L E I N F O}

\section{Article history:}

Received 5 May 2008

Received in revised form 3 October 2008

Accepted 17 January 2009

Available online $\mathrm{xxxx}$

\section{Keywords:}

Strong embedded discontinuity

Discrete cracking

\begin{abstract}
A B S T R A C T
In this paper, strong discontinuities are embedded in finite elements to describe fracture in quasi-brittle materials. A new formulation is presented in which global nodes are introduced along the crack path. The displacement jumps are transferred to the element nodes as a rigid body motion. This approach is compared to the discrete approach, in which interface elements are inserted to model discontinuities. The adopted embedded discontinuities and the interface elements share similar kinematics as well as the same numerical integration schemes. Thus, the present formulation is obtained within the framework of a discrete approach and this is why it is called the discrete strong discontinuity approach (DSDA). Numerical tests are considered, namely a shear band, a mode-I and a mixed-mode fracture examples and a failure test of a RC beam externally reinforced with a steel sheet. Results are compared with those obtained from analyses using interface elements and with experimental results. Finally, conclusions are drawn with respect to mesh independence and robustness of the method.
\end{abstract}

(c) 2009 Elsevier Ltd. All rights reserved.

\section{Introduction}

The use of the cohesive crack approach and non-linear fracture mechanics concepts [1] opened the possibility of accurately modelling fracture behaviour of quasi-brittle materials, such as concrete, masonry and mortar. This cohesive approach is also known as the discrete crack approach, in which it is assumed that microcracking localizes into a surface of discontinuity, designated as a fictitious crack, modelled by interface elements in the finite element mesh. In the discrete crack approach some numerical problems exist, related to the fact that the discontinuities must evolve along finite element boundaries, although the crack path is not usually known a priori. There were two major attempts to circumvent this difficulty at an early stage: (i) the first made use of a remeshing algorithm which enabled the element boundaries to approximate the true crack path [2-4] and (ii) in the second, the remeshing was not performed and the properties of the crack path were projected into the fixed element boundaries [5,6].

More recently, discontinuities were embedded within finite elements, giving rise to: (i) weak discontinuity formulations and (ii) strong discontinuity formulations. In the former case, the discontinuities are modelled as finite width bands and the displacement field remains continuous [7], whereas in the second case, the kinematics of a true discontinuous displacement field is approximated. Many examples of the strong discontinuity approach can be found in literature [8-22], which are compared and discussed in [23]. Moreover, towards a unified view of both weak and strong discontinuities, Oliver et al. [24,25] introduced a continuum strong discontinuity approach (CSDA), in which a strong discontinuity is consistently obtained from a weak discontinuity when the crack band width tends to zero. In all these works, constant displacement jumps are adopted within each parent finite element, the parent element is a constant strain triangle, a local formulation at element level is

\footnotetext{
* Corresponding author. Tel.: +351 239797256; fax: +351 239797259.

E-mail addresses: dcosta@dec.uc.pt (D. Dias-da-Costa), alfaiate@civil.ist.utl.pt (J. Alfaiate), L.J.Sluys@tudelft.nl (L.J. Sluys), ejulio@dec.uc.pt (E. Júlio).
} 
introduced and a non-symmetric variational formulation is built. As a consequence, the jumps are not continuous across element boundaries and stress locking may occur, depending on the relative position of the discontinuity with respect to the element edges $[23,17,26]$. Oliver et al. [24,25], developed an algorithm aiming to eliminate stress locking. In the work presented in Sancho et al. [27], a different approach is introduced where crack path continuity is not enforced and the element is always crossed in the best possible way, thus also avoiding locking. Moreover, this formulation is built upon a consistent weak symmetric formulation, although the usual non-symmetric one is also adopted in [28].

In a different approach, the possibility of interpolating a field over a body using partitions of unity is explored. This approximation was introduced in the generalized finite element method (GFEM), also known as the extended finite element method (X-FEM) and the partition of unity method (PUM) [29-34]. In this method, the jumps are modelled by additional global degrees of freedom located at the standard element nodes. As a consequence, continuous jumps across element boundaries can be obtained. This formulation is more general than the previous embedded ones since, in principle, any parent element can be adopted. However, each element in the vicinity of the crack path must contain the double of the usual number of nodes. As a consequence, the total number of degrees of freedom increases rapidly. Furthermore, in this formulation the concept of embedded discontinuities is no longer addressed.

In Alfaiate et al. [35], higher order discontinuities are embedded in finite elements: two new nodes are located along the discontinuity originating linear displacement jumps. Both local and global implementations of this model can be performed. In the first case the benefits consist of keeping a constant stiffness matrix bandwidth, although the jumps remain discontinuous across element boundaries. Bolzon [36] and Linder and Armero [26] also introduced linear interpolation for the jumps using a local formulation. In the second case, the additional degrees of freedom are global and the jumps are continuous across element boundaries but the bandwidth of the stiffness matrix increases upon crack propagation.

In this paper, a new strong embedded discontinuity formulation is presented using higher order discontinuities embedded in finite elements. As in the GFEM/XFEM, any parent element can be used but the transmission of the jumps into the total element displacements consists of a rigid body motion. As a consequence, the orthogonality condition presented in Simo et al. [10] is fulfilled, since the enhanced strains are zero. Furthermore, it is shown below that this approach is inspired by the discrete-interface approach since: (i) it is developed within the scope of a pure discrete crack approach, in which the discontinuity behaviour is defined separately from the bulk behaviour and (ii) it inherits the same modelling of the discontinuity which is considered an internal interface of the element. This is why the method is called hereafter the discrete strong discontinuity approach (DSDA).

In Section 2, the kinematics of a body crossed by a discontinuity is presented. Several possibilities are exploited and discussed, corresponding to different ways of transferring the displacement jumps to the regular nodes. Next, in Section 3, a review of the variational formulation is presented as a common basis for both the current approach and the discrete-interface approach. In Section 4, the finite element approximation is introduced. A global formulation is adopted, which is consistent with the enforcement of the continuity of the crack path, leading to continuous displacement jumps across element boundaries. In particular, the numerical integration of the discontinuity is discussed. Due to the similarity between the embedded discontinuities in this model and the interface elements in the discrete-interface approach, some numerical issues from the latter are also inherited. In Section 4.5 and in Appendix A, these issues are addressed. In Section 6, numerical examples are presented and the corresponding results are compared to experimental results and to the results obtained with the discrete-interface approach.

\section{Kinematics of a strong discontinuity}

In this section, the kinematics of the displacement and strain fields of a continuum crossed by an embedded discontinuity is studied using tensor notation.

A strong discontinuity is characterized by a jump on the displacement field, localized at a surface. Consider a domain $\Omega$, with boundary $\partial \Omega$ where a discontinuity surface $\Gamma_{d}$ is supposed to exist (Fig. 1). The external tractions are applied at part $\Gamma_{t}$ of the boundary whereas the displacements are imposed on part $\Gamma_{u}$ of $\partial \Omega$, such that $\Gamma_{t} \cup \Gamma_{u}=\partial \Omega$ and $\Gamma_{t} \cap \Gamma_{u}=\varnothing$.

The total displacement field is the sum of a continuous regular part on $\Omega$, $\hat{\mathbf{u}}$, and a discontinuous part, $\tilde{\mathbf{u}}$, corresponding to the displacement jump $\llbracket \mathbf{u} \rrbracket$, localized at the discontinuity surface $\Gamma_{d}$. The total displacement field $\mathbf{u}(\mathbf{x})$ can be evaluated as:

$$
\mathbf{u}(\mathbf{x})=\hat{\mathbf{u}}(\mathbf{x})+\mathscr{H}_{\Gamma_{d}} \tilde{\mathbf{u}}(\mathbf{x})
$$

where $\mathscr{H}_{\Gamma_{d}}$ is the Heaviside function at the discontinuity $\Gamma_{d}$,

$$
\mathscr{H}_{\Gamma_{d}}=\left\{\begin{array}{ll}
1 & \text { if } \mathbf{x} \in \Omega^{+} \\
0 & \text { otherwise }
\end{array} .\right.
$$

In Eq. (1), the additional displacement field $\tilde{\mathbf{u}}$ is such that:

$$
\tilde{\mathbf{u}}_{\mid \Gamma_{d}}=\mathbf{u}^{+}-\mathbf{u}^{-}=\llbracket \mathbf{u} \rrbracket .
$$

The strain field obtained from the continuous part of the displacement field is:

$$
\hat{\boldsymbol{\epsilon}}=\nabla^{\mathrm{s}} \hat{\mathbf{u}} \text { in } \Omega \backslash \Gamma_{d},
$$



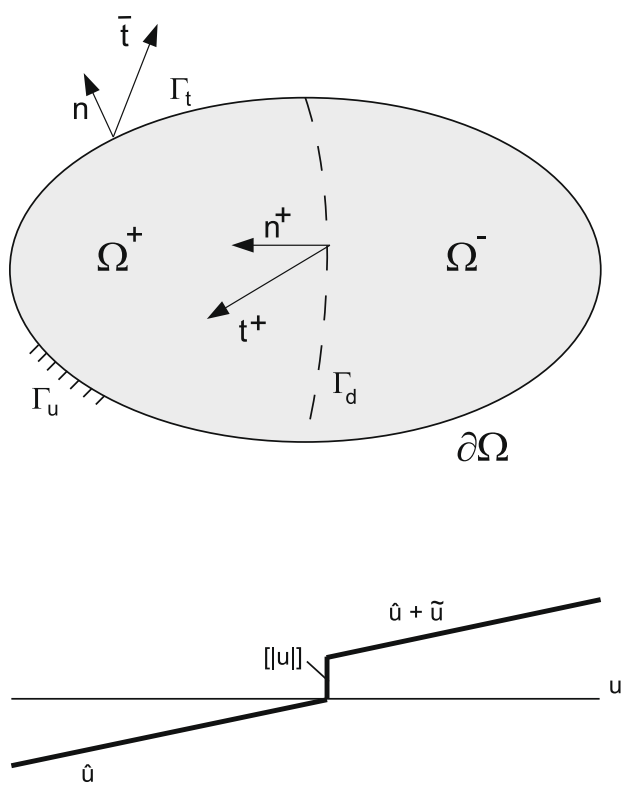

Fig. 1. Domain $\Omega$ crossed by a discontinuity surface $\Gamma_{d}$.

where $(\cdot)^{s}$ refers to the symmetric part of $(\cdot)$. The total strain in the body is given by:

$$
\boldsymbol{\epsilon}=\nabla^{\mathrm{s}} \mathbf{u}=\underbrace{\boldsymbol{\nabla}^{\mathrm{s}} \hat{\mathbf{u}}+\mathscr{H}_{\Gamma_{d}}\left(\nabla^{\mathrm{s}} \llbracket \mathbf{u} \rrbracket\right)}_{\text {bounded }}+\underbrace{\delta_{\Gamma_{d}}(\llbracket \mathbf{u} \rrbracket \otimes \mathbf{n})^{\mathrm{s}}}_{\text {unbounded }} \text { in } \Omega,
$$

where $\otimes$ denotes a dyadic product and $\delta_{\Gamma_{d}}$ is the Dirac delta-function along surface $\Gamma_{d}$. Both the displacement field and the strain field are continuous in $\Omega^{-}$and $\Omega^{+}$, since the unbounded term in Eq. (5) vanishes in $\Omega \backslash \Gamma_{d}=\Omega^{-} \cup \Omega^{+}$.

\section{Variational formulation}

The governing field equations are imposed separately in $\Omega \backslash \Gamma_{d}$ and on $\Gamma_{d}$. Together with the boundary conditions, these can be expressed as (see Fig. 1):

$$
\begin{aligned}
& \nabla \cdot \boldsymbol{\sigma}+\mathbf{b}=\mathbf{0} \text { in } \Omega \backslash \Gamma_{d}, \\
& \boldsymbol{\epsilon}=\nabla^{\mathrm{s}} \mathbf{u} \text { in } \Omega \backslash \Gamma_{d}, \\
& \boldsymbol{\sigma}=\boldsymbol{\sigma}(\boldsymbol{\epsilon}) \text { in } \Omega \backslash \Gamma_{d}, \\
& \mathbf{u}=\overline{\mathbf{u}} \text { at } \Gamma_{u}, \\
& \boldsymbol{\sigma} \cdot \mathbf{n}=\overline{\mathbf{t}} \text { at } \Gamma_{t}, \\
& \boldsymbol{\sigma}^{+} \cdot \mathbf{n}^{+}=\mathbf{t}^{+} \text {at } \Gamma_{d}, \\
& \boldsymbol{\sigma}^{-} \cdot \mathbf{n}^{-}=\mathbf{t}^{-} \text {at } \Gamma_{d}, \\
& \mathbf{t}^{+}=-\mathbf{t}^{-}=\mathbf{t} \text { at } \Gamma_{d},
\end{aligned}
$$

where $\mathbf{b}$ are the body forces and $\overline{\mathbf{u}}$ and $\overline{\mathbf{t}}$ are the prescribed displacements and tractions at the boundary, respectively. Eqs. (6), (9), (10) are, respectively: the equilibrium equations, the essential, and the natural boundary conditions. Eqs. (7) are the strain displacement relations and Eqs. (8) are the constitutive laws. Eqs. (11) and (12) enforce traction continuity across the discontinuity surface $\Gamma_{d}$, where the tractions are denoted by $\mathbf{t}$. As depicted in Fig. $1, \mathbf{n}^{+}=-\mathbf{n}^{-}$, is the inward normal of $\Omega^{+}$ and $\mathbf{t}^{+}=-\mathbf{t}^{-}$denotes the traction vector acting on $\Omega^{+}$.

In Alfaiate et al. [35], the variational formulation is reviewed as a common basis for various approaches. For instance in Simo et al. [10] and in Lofti and Shing [11], the extension of the three-field Hu-Washizu variational statements to a body containing an internal discontinuity surface is considered [37]. In [10], a non-symmetric formulation is obtained due to the fact that the traction continuity condition is imposed in a strong form, although in average. In [11], a consistent symmetric weak formulation is introduced, in which the traction continuity is enforced, as usual, in the weak sense. The extension of the three-field Hu-Washizu variational statements is the most general assumption since it is possible to consider $\mathbf{u}, \boldsymbol{\epsilon}, \boldsymbol{\sigma}, \llbracket \mathbf{u} \rrbracket$ as independent unknown fields. This possibility was explored in Lofti and Shing [11], where mixed finite elements were proposed to approximate the independent unknown fields.

In the adopted approach, a consistent symmetric weak form is adopted, in which Eqs. (7) are assumed to be satisfied according to (5). This is usually the case for both the GFEM and the discrete-interface approach. For instance, in the first case, 
Simone [38] considers the discontinuity as an internal boundary and separately imposes the principle of virtual work on $\Omega$ and $\Omega^{+}$, whereas Wells [39] uses the properties of the dirac delta-function to obtain the energy in the discontinuity.

Here, the variational formulation is presented in the same format which is adopted in the discrete-interface approach. Considering the principle of virtual work, taking the strain energy $\int_{\Omega \backslash \Gamma_{d}}\left(\nabla^{\mathrm{s}} \delta \mathbf{u}\right): \sigma d \Omega$ and the external work $\int_{\Omega \backslash \Gamma_{d}} \delta \mathbf{u} \cdot \mathbf{b} d \Omega+\int_{\Gamma_{t}} \delta \mathbf{u} \cdot \overline{\mathbf{t}} d \Gamma$, which are the usual terms in a continuum approach, and adding the term corresponding to the work done in the discontinuity $\int_{\Gamma_{d}} \delta \llbracket \mathbf{u} \rrbracket \cdot \mathbf{t}^{+} d \Gamma$, it is obtained:

$$
-\int_{\Omega \backslash \Gamma_{d}}\left(\nabla^{\mathrm{s}} \delta \mathbf{u}\right): \boldsymbol{\sigma} d \Omega+\int_{\Omega \backslash \Gamma_{d}} \delta \mathbf{u} \cdot \mathbf{b} d \Omega+\int_{\Gamma_{\mathrm{t}}} \delta \mathbf{u} \cdot \overline{\mathbf{t}} d \Gamma+\int_{\Gamma_{d}} \delta \llbracket \mathbf{u} \rrbracket \cdot \mathbf{t}^{+} d \Gamma=0,
$$

where (.) and (:) refer to single and double contractions, respectively. This variational formulation was presented in Malvern [40] and can be applied to all strong discontinuity formulations mentioned above.

According to Eq. (1) $\delta \mathbf{u}=\delta \hat{\mathbf{u}}+\mathscr{H}_{\Gamma_{d}} \delta \tilde{\mathbf{u}}$, and considering nonzero variations of $\delta \tilde{\mathbf{u}}$ and $\delta \hat{\mathbf{u}}$, two variational statements are obtained:

$$
\begin{aligned}
& -\int_{\Omega \backslash \Gamma_{d}}\left(\nabla^{\mathrm{s}} \delta \hat{\mathbf{u}}\right): \boldsymbol{\sigma}(\hat{\boldsymbol{\epsilon}}) d \Omega+\int_{\Omega \backslash \Gamma_{d}} \delta \hat{\mathbf{u}} \cdot \mathbf{b} d \Omega+\int_{\Gamma_{t}} \delta \hat{\mathbf{u}} \cdot \overline{\mathbf{t}} d \Gamma=0, \\
& -\int_{\Omega^{+}}\left(\nabla^{\mathrm{s}} \delta \tilde{\mathbf{u}}\right): \boldsymbol{\sigma}(\hat{\boldsymbol{\epsilon}}) d \Omega+\int_{\Omega^{+}} \delta \tilde{\mathbf{u}} \cdot \mathbf{b} d \Omega+\int_{\Gamma_{t}} \delta \tilde{\mathbf{u}} \cdot \overline{\mathbf{t}} d \Gamma+\int_{\Gamma_{d}} \delta\left[\mathbf{u} \rrbracket \cdot \mathbf{t}^{+} d \Gamma=0 .\right.
\end{aligned}
$$

Eq. (15) is the result of the Principle of Virtual Work applied to the bulk, whereas Eq. (16) corresponds to the work done by the additional displacement field $\tilde{\mathbf{u}}$ in $\Omega \backslash \Gamma_{d}$ as well as in the discontinuity $\Gamma_{d}$.

\section{Finite element approximation}

Consider a finite element discretisation of the 2D domain $\Omega$. Assume that one element is crossed by a straight discontinuity $\Gamma_{d}$, which divides $\Omega$ in two sub-domains $\Omega^{+}$and $\Omega^{-}$. A local frame $(s, n)$ is introduced such that $s(\mathbf{x})$ is aligned with $\Gamma_{d}$ and $n$ is the normal to the discontinuity (Figs. 2 and 3 ).

\subsection{Non-homogeneous jumps}

From Eq. (1), it can be assumed, by simplicity, that the jump $\llbracket \mathbf{u} \rrbracket$ is a linear function of $s$, where:

$$
\llbracket \mathbf{u} \rrbracket=\llbracket \mathbf{u}(s(\mathbf{x})) \rrbracket=\tilde{\mathbf{u}}_{\mid \Gamma_{d}} .
$$

In Figs. 2 and 3, where $\hat{\mathbf{u}}$ is neglected for clarity, the total displacement field is depicted in two different situations: in Fig. 2 the discontinuity opens in normal direction only, whereas in Fig. 3 the discontinuity represents a shear band undergoing sliding displacements.

If the jump is assumed to be constant across $\Gamma_{d}$, it is sufficient to adopt one internal node to represent function $\llbracket \mathbf{u} \rrbracket$. However, if higher order functions are considered to approximate additional displacements, two more nodes are required for a linear function, marked in white in Figs. 2 and 3, three more nodes for a quadratic function, and so forth. In the example above, the additional two nodes ( $i$ and $j$ ) are located at the intersection of $\Gamma_{d}$ with the edges of the element.

In matrix form, for each finite element $e$ with $n$ nodes, the following approximation of the displacement field is adopted:

$$
\begin{aligned}
& \hat{\mathbf{u}}^{e}=\mathbf{N}^{e}(\mathbf{x}) \hat{\mathbf{a}}^{e} \text { in } \Omega^{e} \backslash \Gamma_{d}^{e}, \\
& \llbracket \mathbf{u} \rrbracket^{e}=\mathbf{N}_{w}^{e}[S(\mathbf{x})] \mathbf{w}^{e} \text { at } \Gamma_{d}^{e},
\end{aligned}
$$
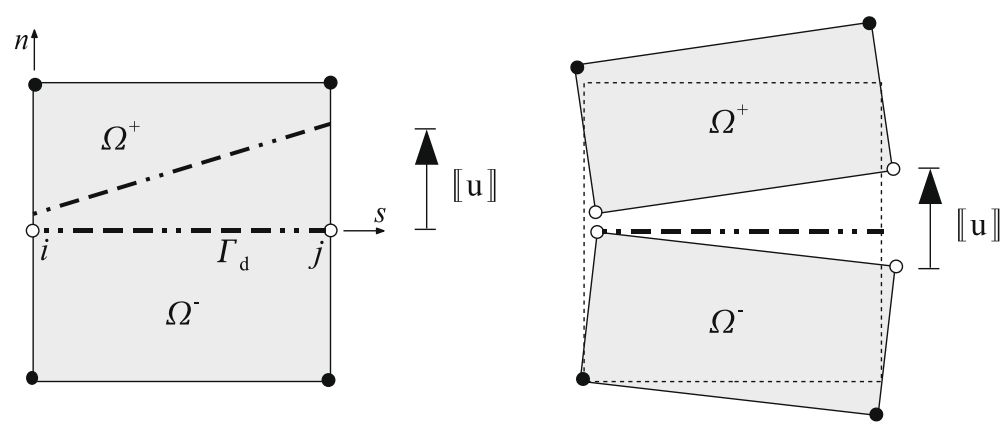

Fig. 2. Normal jump displacement in a four node element crossed by a discontinuity. 

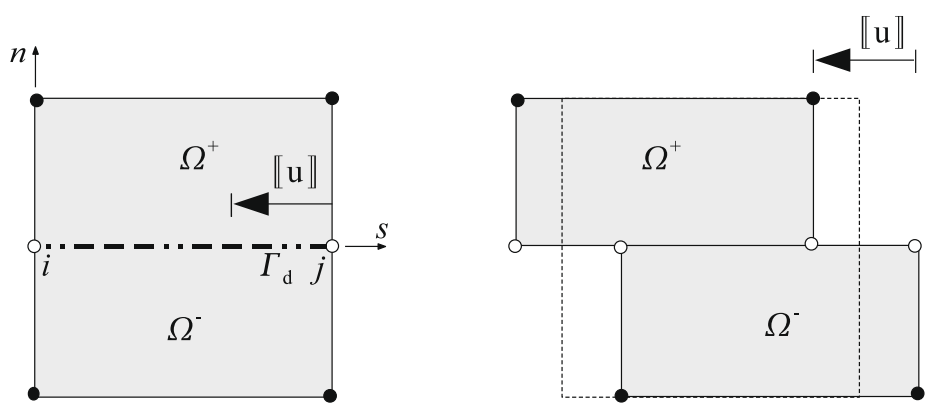

Fig. 3. Shear band in a four node element crossed by a discontinuity.

where $\mathbf{N}^{e}$ contains the usual element shape functions; $\hat{\mathbf{a}}^{e}$ are the nodal degrees of freedom associated with $\hat{\mathbf{u}}^{e} ; \mathbf{N}_{w}^{e}$ are the shape functions used to approximate the jumps $\llbracket \mathbf{u} \rrbracket^{e}$ which, in turn, are approximated by the degrees of freedom $\mathbf{w}^{e}$ measured at nodes $i$ and $j$. If the number of nodes in Eqs. (18), used to approximate the jumps, is $n_{w}, \mathbf{N}_{w}^{e}$ is a $\left(2 \times n_{w}\right)$ matrix; if $n_{w}=1, \mathbf{N}_{w}^{e}$ is the unit matrix; if $n_{w}=2, \mathbf{N}_{w}^{e}$ contains linear shape functions, and so on.

\subsection{Orthogonality}

In the works adopting the embedded discontinuity approach presented in Alfaiate et al. [35] and Linder and Armero [26], the total strain field in $\Omega^{e} \backslash \Gamma_{d}^{e}$ is different from the regular strain field due to the regular displacements, i.e.,

$$
\boldsymbol{\epsilon}^{e} \neq \hat{\boldsymbol{\epsilon}}^{e}=\nabla^{\mathrm{s}} \hat{\mathbf{u}}^{e} \text { in } \Omega^{e} \backslash \Gamma_{d}^{e}
$$

In Simo and Rifai [41], it is assumed that $S^{h}$ and $\tilde{\zeta}^{h}$ are $L_{2}$ orthogonal, where $S^{h}$ and $\tilde{\zeta}^{h}$ are the admissible stress space and the admissible enhanced strain space, respectively. As a result, the work done by the stresses on the enhanced strains within the parent element is null. Applying this orthogonality condition to $\Omega^{e} \backslash \Gamma_{d}$, gives:

$$
\int_{\Omega^{e} \backslash \Gamma_{d}}\left(\nabla^{S} \tilde{\mathbf{u}}^{e}\right)^{T}: \sigma^{e} d \Omega=0
$$

As it will become clear in Section 4.4, this condition is satisfied in all formulations adopting embedded discontinuities with constant jumps, namely in the works from Simo et al. [10], Lofti and Shing [11], Armero and Callari [12], Oliver [14,15], Jirásek and Zimmermann [17,18], Borja [20], Wells and Sluys [21], Alfaiate et al. [22].

A physical interpretation of Eq. (20) can be given in the sense that the stresses in the element are only due to the regular part of the strain field, $\boldsymbol{\sigma}=\boldsymbol{\sigma}(\hat{\boldsymbol{\epsilon}})$. This result, which is assumed in the variational formulation (see Eqs. (15) and (16)) and in the works presented in Alfaiate et al. [22,35], is also in agreement with the bounded nature of the stress field as stated by Oliver [14]. However, if inequality (19) holds, the orthogonality condition (20) is violated. In the present formulation, Eq. (20) is enforced exactly, by imposing that the enhanced displacements $\tilde{\mathbf{u}}^{e}$ induce a null enhanced strain field [42]:

$$
\tilde{\boldsymbol{\epsilon}}^{e}=\nabla^{\mathrm{s}} \tilde{\mathbf{u}}^{e}=\mathbf{0} \text { in } \Omega^{e} \backslash \Gamma_{d} .
$$

Consequently, the additional displacement field $\tilde{\mathbf{u}}^{e}$ corresponds to a rigid body motion from $\Omega^{+}$with respect to $\Omega^{-}$:

$$
\tilde{\mathbf{u}}^{e}=\mathbf{M}_{w}^{e}(\mathbf{x}) \mathbf{w}^{e} .
$$

In Eq. (22), $\mathbf{M}_{w}^{e}$ is a $\left(2 \times n_{w}\right)$ matrix which generates a rigid body motion of $\Omega^{e+}$ relative to $\Omega^{e_{-}}$. It is given by:

$$
\mathbf{M}_{w}^{e T}=\left[\begin{array}{cc}
1-\frac{\left(\left(x_{2}-x_{2}^{i}\right) \sin \alpha\right)}{l_{d}} & \frac{\left(x_{1}-x_{1}^{i}\right) \sin \alpha}{l_{d}} \\
\frac{\left(x_{2}-x_{2}^{i}\right) \cos \alpha}{l_{d}} & 1-\frac{\left(x_{1}-x_{1}^{i}\right) \cos \alpha}{l_{d}} \\
\frac{\left(x_{2}-x_{2}^{i}\right) \sin \alpha}{l_{d}} & -\frac{\left(x_{1}-x_{1}^{i}\right) \sin \alpha}{l_{d}} \\
\frac{-\left(x_{2}-x_{2}^{i}\right) \cos \alpha}{l_{d}} & \frac{\left(x_{1}-x_{1}^{i}\right) \cos \alpha}{l_{d}}
\end{array}\right],
$$

where $l_{d}$ is the length of the discontinuity $\Gamma_{d}^{e}$ and $\alpha$ is the angle between coordinates $s$ and $x_{1}$ (see Fig. 4).

It should be noted that, independently of the parent element chosen and for a straight discontinuity $\Gamma_{d}^{e}$, the rigid body motions of $\Omega^{e+}$ and $\Omega^{e-}$ are totally defined by only three additional independent degrees of freedom. The corresponding shear jump component along the discontinuity is therefore constant, which gives rise to the additional linear constraint equation: 

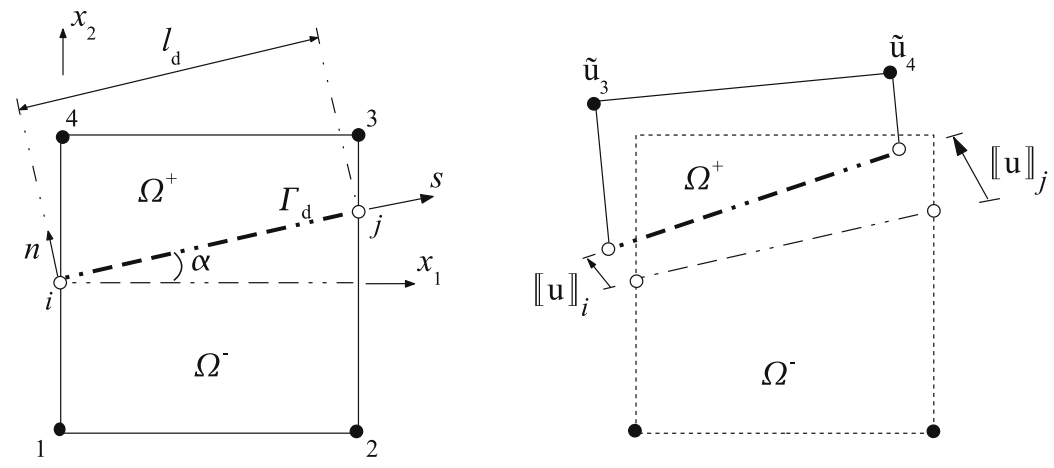

Fig. 4. Rigid body motion $\tilde{\mathbf{u}}$ due to discontinuity jumps $\llbracket \mathbf{u} \rrbracket$.

$$
w_{s}^{i}=w_{s}^{j},
$$

where $w_{s}^{i}$ and $w_{s}^{j}$ are the tangential jump components obtained at discontinuity nodes $i$ and $j$, respectively. In this case the following equality is obtained:

$$
\mathbf{u}^{e+}-\mathbf{u}^{e-}=\left.\mathbf{M}_{w}^{e}\right|_{\Gamma_{d}^{e}} \mathbf{W}^{e}=\llbracket \mathbf{u} \rrbracket^{e}=\mathbf{N}_{w}^{e} \mathbf{w}^{e} .
$$

\subsection{A discrete crack approach}

The orthogonality condition can also be viewed as a direct consequence of the discrete-interface approach. In fact, it is shown in Section 4.4 that, similar to the latter, it is also possible in the DSDA to decouple the work done in the continuum from the energy spent in the discontinuity. This is in accordance with the basic hypotheses of the discrete crack approach and how it relates to physical reality. For instance, considering a concrete specimen submitted to tension (Fig. 5), in a discrete crack approach it is assumed that:

(1) Microcracking develops whenever the tensile strength is reached.

(2) Microcracking is localized in a zero width surface of discontinuity, here designated as strong discontinuity.

(3) This strong discontinuity evolves according to a localized stress-jump relationship undergoing softening.

(4) At the same time, the bulk unloads due to the traction continuity condition.

(5) The total elongation in the specimen is the sum of the continuous part plus the jump, which is transmitted as a rigid body motion.

There are two statements above which provide the link between the bulk and the discontinuity: (1) and (4). The first could be rewritten in a more general form such as: microcracking develops at the onset of strain localization. In the Continuum Strong Discontinuity Approach, this condition is automatically satisfied through the definition of the acoustic tensor, as presented in Oliver et al. [25]. As it is shown in Section 5, both in the discrete-interface approach and in the DSDA this condition is replaced by an initiation criterion, which must be defined separately. Statement (4) is common to all formulations since it is an equilibrium condition. In the first embedded discontinuity formulations this condition was imposed in a strong form, although in average. In the discrete-interface approach, in the DSDA, in other embedded formulations such as the works presented in Sancho et al. [27] and Lofti and Shing [11], as well as in the GFEM/XFEM, traction continuity is imposed in a weak form. The numerical implementation of the DSDA is discussed in Section 5.

Thus, in the discrete crack approach, although continuum and non-continuum can be characterized through different material types and constitutive formats as stated in Oliver et al. [25], these are not completely independent from each other. Apart from the usual traction continuity condition, the link between continuum and discontinuum is introduced through the definition of an initiation criterion, given in Section 5.

\subsection{Discretization}

The displacement field in each element is given by:

$$
\begin{aligned}
& \mathbf{u}^{e}=\mathbf{N}^{e}(\mathbf{x})\left(\hat{\mathbf{a}}^{e}+\mathscr{H}_{\Gamma_{d}} \mathbf{M}_{w}^{e k} \mathbf{w}^{e}\right) \text { if } \mathbf{x} \in \Omega^{e} \backslash \Gamma_{d} \\
& \llbracket \mathbf{u} \rrbracket^{e}=\mathbf{N}_{w}^{e}[s(\mathbf{x})] \mathbf{w}^{e} \text { at } \Gamma_{d}^{e} .
\end{aligned}
$$



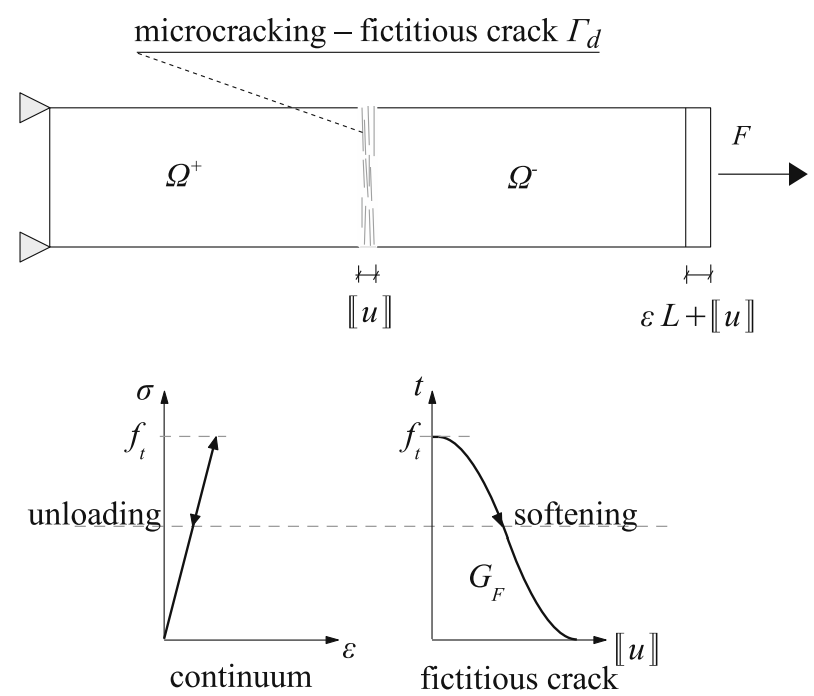

Fig. 5. Specimen submitted to tension-the discrete crack approach.

were $\mathbf{M}_{w}^{e k}$ is formed by the evaluation of the Eq. (23) in each of the $n$ nodes of the element.

The regular strain field is approximated by:

$$
\hat{\boldsymbol{\epsilon}}^{e}=\mathbf{L} \mathbf{N}^{e}(\mathbf{x}) \hat{\mathbf{a}}^{e}=\mathbf{B}^{e}(\mathbf{x}) \hat{\mathbf{a}}^{e},
$$

where $\mathbf{L}$ is the usual differential operator.

Let the unknowns be $\mathbf{a}^{e}$ and $\mathbf{w}^{e}$, where $\mathbf{a}^{e}$ are the total nodal displacements obtained at the regular nodes $k$ :

$$
\mathbf{a}^{e}=\hat{\mathbf{a}}^{e}+\mathbf{H}_{\Gamma_{d}}^{e} \tilde{\mathbf{a}}^{e},
$$

where $\mathbf{H}_{\Gamma_{d}}^{e}=\mathscr{H}_{\Gamma_{d}} \mathbf{I}$, with $\mathbf{I}$ standing for the $(2 n \times 2 n)$ identity matrix, and:

$$
\tilde{\mathbf{a}}^{e}=\mathbf{M}_{w}^{e k} \mathbf{w}^{e} .
$$

The regular strain field is given by:

$$
\hat{\epsilon}^{e}=\mathbf{B}^{e}\left(\mathbf{a}^{e}-\mathbf{H}_{\Gamma_{d}}^{e} \mathbf{M}_{w}^{e k} \mathbf{w}^{e}\right) .
$$

The incremental stress field is:

$$
d \boldsymbol{\sigma}^{e}=\mathbf{D}^{e} \mathbf{B}^{e}\left(d \mathbf{a}^{e}-\mathbf{H}_{\Gamma_{d}}^{e} \mathbf{M}_{w}^{e k} d \mathbf{w}^{e}\right) .
$$

As in the discrete-interface approach, the tractions are obtained from the traction-jump law at the discontinuity. In incremental format this gives:

$$
d \mathbf{t}^{e}=\mathbf{T}^{e} d \llbracket \mathbf{u} \rrbracket^{e}=\mathbf{T}^{e} \mathbf{N}_{w}^{e} d \mathbf{w}^{e} \text { at } \Gamma_{d}^{e} .
$$

Discretising Eqs. (15) and (16) by means of the field approximations given in Eqs. (18), (31) and (32), the following uncoupled system of equations can be obtained:

$$
\begin{aligned}
& \mathbf{K}_{a a}^{e} d \hat{\mathbf{a}}^{e}=d \mathbf{f}_{\text {ext }}^{e}, \\
& \mathbf{K}_{d}^{e} d \mathbf{w}^{e}=d \mathbf{f}_{w, e x t}^{e},
\end{aligned}
$$

where

$$
\begin{aligned}
d \hat{\mathbf{a}}^{e} & =d \mathbf{a}^{e}-\mathbf{H}_{\Gamma_{d}}^{e} \mathbf{M}_{w}^{e k} d \mathbf{w}^{e}, \\
\mathbf{K}_{a a}^{e} & =\int_{\Omega^{e}} \mathbf{B}^{e T} \mathbf{D}^{e} \mathbf{B}^{e} d \Omega, \\
\mathbf{K}_{d}^{e} & =\int_{\Gamma_{d}^{e}} \mathbf{N}_{w e}^{T} \mathbf{T}^{e} \mathbf{N}_{w}^{e} d \Gamma,
\end{aligned}
$$


and

$$
\begin{aligned}
& d \mathbf{f}_{\text {ext }}^{e}=\int_{\Omega^{e}} \mathbf{N}^{e T} d \mathbf{b}^{e} d \Omega+\int_{\Gamma_{t}^{e}} \mathbf{N}^{e T} d \overline{\mathbf{t}}^{e} d \Gamma, \\
& d \mathbf{f}_{w, e x t}^{e}=\int_{\Omega^{e+}}\left(\mathbf{M}_{w}^{e k}\right)^{T} \mathbf{N}^{e T} d \mathbf{b}^{e} d \Omega+\int_{\Gamma_{t}^{e^{+}}}\left(\mathbf{M}_{w}^{e k}\right)^{T} \mathbf{N}^{e T} d \overline{\mathbf{t}}^{e} d \Gamma .
\end{aligned}
$$

Note that, in Eq. (34), the terms related to the gradient of the enhanced strain field are absent, since $\nabla^{s} \delta \tilde{\mathbf{u}}^{e}=\mathbf{0}$.

It should be noted that a system of equations similar to the ones adopted in the discrete-interface approach is obtained, in which Eq. (33) takes into account the bulk behaviour, modelled with the usual finite elements, whereas Eq. (34) takes into account the discrete law, modelled using the interface elements. However, in the embedded approach, instead of $\hat{\mathbf{a}}^{e}$, the unknowns must be the total degrees of freedom $\mathbf{a}^{e}$ of the parent element, which include both the regular degrees of freedom $\hat{\mathbf{a}}^{e}$ related to the bulk, and the enhanced degrees of freedom $\tilde{\mathbf{a}}^{e}$, related to the discontinuity jumps. As a consequence, Eqs. (33) and (34), given in an uncoupled form, must be rewritten in a coupled form.

Replacing dâ from Eq. (35) in Eq. (33), gives:

$$
\left[\begin{array}{cc}
\mathbf{K}_{a a}^{e} & \mathbf{0} \\
\mathbf{0} & \mathbf{K}_{d}^{e}
\end{array}\right]\left\{\begin{array}{c}
d \mathbf{a}^{e}-\mathbf{H}_{\Gamma_{d}}^{e} \mathbf{M}_{w}^{e k} d \mathbf{w}^{e} \\
d \mathbf{w}^{e}
\end{array}\right\}=\left\{\begin{array}{c}
d \mathbf{f}_{e x t}^{e} \\
d \mathbf{f}_{w, e x t}^{e}
\end{array}\right\}
$$

or equivalently

$$
\left[\begin{array}{cc}
\mathbf{K}_{a a}^{e} & -\mathbf{K}_{a a}^{e} \mathbf{H}_{\Gamma_{d}}^{e} \mathbf{M}_{w}^{e k} \\
\mathbf{0} & \mathbf{K}_{d}^{e}
\end{array}\right]\left\{\begin{array}{c}
d \mathbf{a}^{e} \\
d \mathbf{w}^{e}
\end{array}\right\}=\left\{\begin{array}{c}
d \mathbf{f}_{e x t}^{e} \\
d \mathbf{f}_{w, e x t}^{e}
\end{array}\right\} .
$$

Eq. (41) relates the total nodal displacements to the nodal jumps. A symmetric form can be obtained by the following algebraic manipulation:

$$
\left[\begin{array}{cc}
\mathbf{I} & \mathbf{0} \\
-\left(\mathbf{H}_{\Gamma_{d}}^{e} \mathbf{M}_{w}^{e k}\right)^{T} & \mathbf{I}
\end{array}\right]\left[\begin{array}{cc}
\mathbf{K}_{a a}^{e} & -\mathbf{K}_{a a}^{e} \mathbf{H}_{\Gamma_{d}}^{e} \mathbf{M}_{w}^{e k} \\
\mathbf{0} & \mathbf{K}_{d}^{e}
\end{array}\right]\left\{\begin{array}{c}
d \mathbf{a}^{e} \\
d \mathbf{w}^{e}
\end{array}\right\}=\left[\begin{array}{cc}
\mathbf{I} & \mathbf{0} \\
-\left(\mathbf{H}_{\Gamma_{d}}^{e} \mathbf{M}_{w}^{e k}\right)^{T} & \mathbf{I}
\end{array}\right]\left\{\begin{array}{c}
d \mathbf{f}_{e x t}^{e} \\
d \mathbf{f}_{w, e x t}^{e}
\end{array}\right\},
$$

which can be further simplified into:

$$
\left[\begin{array}{cc}
\mathbf{K}_{a a}^{e} & -\mathbf{K}_{a w}^{e} \\
-\mathbf{K}_{w a}^{e} & \mathbf{K}_{w w}^{e}+\mathbf{K}_{d}^{e}
\end{array}\right]\left\{\begin{array}{c}
d \mathbf{a}^{e} \\
d \mathbf{w}^{e}
\end{array}\right\}=\left\{\begin{array}{c}
d \mathbf{f}_{e x t}^{e} \\
d \mathbf{f}_{w, e x t}^{e}-\left(\mathbf{H}_{\Gamma_{d}}^{e} \mathbf{M}_{w}^{e k}\right)^{T} d \mathbf{f}_{\text {ext }}^{e}
\end{array}\right\}
$$

where,

$$
\begin{aligned}
& \mathbf{K}_{a w}^{e}=\int_{\Omega^{e}} \mathbf{B}^{e T} \mathbf{D}^{e} \mathbf{B}_{w}^{e} d \Omega^{e}, \\
& \mathbf{K}_{w a}^{e}=\mathbf{K}_{a w}^{e T}, \\
& \mathbf{K}_{w w}^{e}=\int_{\Omega^{e}} \mathbf{B}_{w}^{e T} \mathbf{D}^{e} \mathbf{B}_{w}^{e} d \Omega^{e},
\end{aligned}
$$

and

$$
\mathbf{B}_{w}^{e}=\mathbf{B}^{e} \mathbf{H}_{\Gamma_{d}}^{e} \mathbf{M}_{w}^{e k}
$$

If the body forces are neglected and the forces are applied at the nodes, then $d \mathbf{f}_{w, \text { ext }}^{e}-\left(\mathbf{H}_{\Gamma_{\mathrm{d}}}^{e} \mathbf{M}_{w}^{e k}\right)^{T} d \mathbf{f}_{\text {ext }}^{e}=\mathbf{0}$ and only the regular nodal forces $d \mathbf{f}_{\text {ext }}^{e}$ need to be computed. In fact, $\left(\mathbf{H}_{\Gamma_{d}}^{e} \mathbf{M}_{w}^{e k}\right)^{T}$ acts as a means of transferring the nodal forces acting at the external element boundaries into the internal boundary $\Gamma_{d}^{e}$, i.e., $d \mathbf{f}_{w, e x t}^{e}=\left(\mathbf{H}_{\Gamma_{d}}^{e} \mathbf{M}_{w}^{e k}\right)^{T} d \mathbf{f}_{\text {ext }}^{e}$.

The additional nodes can be taken as local, like in previous embedded formulations, or as global, similar to the works presented in Alfaiate et al. [35,43]. This option is adopted in this implementation and therefore, for $n_{w} \geqslant 2$, continuity of the jumps at the discontinuities across the element boundaries is automatically enforced.

Finally, as stated in Section 4.2, for a straight discontinuity $\Gamma_{d}^{e}$, the rigid body motions of $\Omega^{e+}$ and $\Omega^{e-}$ is totally defined by only three additional independent degrees of freedom. These degrees of freedom give rise to both: $i$ ) a translation and ii) a rotation. The first case was taken into account in the former embedded formulations, namely in the works presented in Simo et al. [10], Lofti and Shing [11], Armero and Callari [12], Oliver [14,15], Jirásek and Zimmermann [17,18], Borja [20], Wells and Sluys [21], Alfaiate et al. [22], where a constant jump was adopted. Thus, the present formulation can also be considered as a generalization of the former embedded discontinuity models, in which the rigid body rotation is additionally considered. This is also why all these formulations fulfill Simo's orthogonality condition, as mentioned in Section 4.2.

\subsection{Numerical approximation of an embedded discontinuity in the DSDA}

The embedded discontinuity in the DSDA inherits the kinematic properties of interface elements. The presence of oscillations in the stress profile obtained with interfaces was reported in the literature when Gauss quadrature is used 
[44-46,38]. However, when Newton-Cotes scheme is adopted, smoothing of the response is usually observed [45,38]. In fact, Kikuchi and Oden [47] proved that, in two dimensions, both trapezoidal and Simpson rules give rise to accurate linear and quadratic solutions, respectively, assuming that a sufficiently refined mesh is provided. As a consequence, the trapezoidal rule is adopted for the integration of the stiffness matrix of the embedded discontinuities in the DSDA. Further discussion on the integration of the stiffness matrix of the interface elements is presented in Appendix A.

Rots [44] performed a numerical study in order to assess the performance of interface elements in mode-I fracture. The same example is studied here in order to numerically verify the efficiency of the DSDA in avoiding spurious stress oscillations. These oscillations can also be found in other formulations, for instance GFEM based, which, according to [38], should not be used with a dummy stiffness.

Fig. 6 presents the structural scheme for the notched beam used in [44], with the following bulk material properties: Young modulus $E=20 \mathrm{GPa}$ and Poisson ratio $v=0.2$. The discontinuity constitutive law is linear elastic:

$$
\mathbf{T}^{e}=\left[\begin{array}{cc}
k_{n} & 0 \\
0 & k_{s}
\end{array}\right]
$$

where different normal stiffness parameters $k_{n}$ are used: $10^{3}, 10^{4}, 10^{5}$ and $10^{8} \mathrm{MPa} / \mathrm{mm}$. The notch is simulated with null normal stiffness, whereas the shear stiffness, $k_{s}$, is unimportant, since no shear is present along the symmetry line.

The beam is subjected to a plane stress state and a load of $1 \mathrm{kN}$. Two meshes are studied: a structured and a unstructured mesh (see Fig. 7). In both cases, bilinear finite elements are used. In Fig. 7a a discontinuity length $l_{d}$ equal to $5 \mathrm{~mm}$ is adopted, whereas in Fig. 7b the mean value of $l_{d}$ is $5.7 \mathrm{~mm}$.

In Fig. 8 it is shown that the use of the Newton-Cotes/Lobatto scheme with the DSDA effectively avoids traction oscillations. For the penalization range tested, the results are almost independent of the applied penalty. In Fig. 8, the differences found in the smoothness of the traction profiles are due to the different mesh discretization.

Under mode-II fracture, the connection between the bulk and the discontinuity is not enforced at the discontinuity nodes. Moreover, since the trapezoidal rule induces independent weights between the integration points, the transmission of the

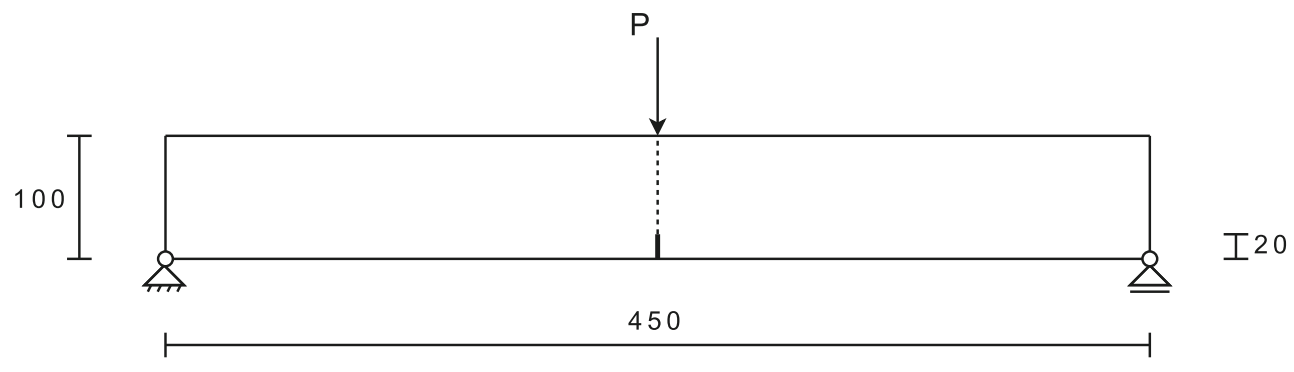

Fig. 6. Notched beam with $100 \mathrm{~mm}$ thickness (all dimensions in $\mathrm{mm}$ ).
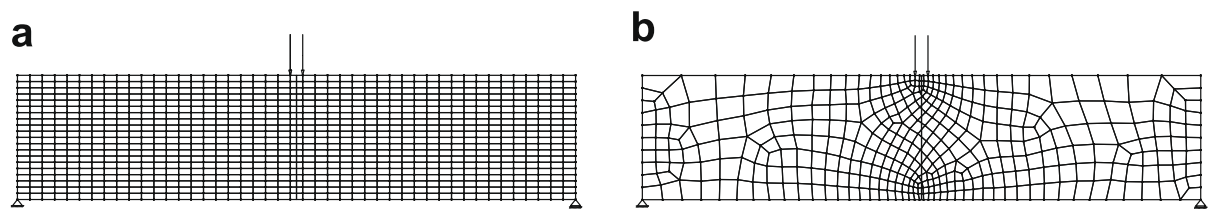

Fig. 7. Mesh used for the DSDA numerical tests: (a) structured mesh; and (b) unstructured mesh.
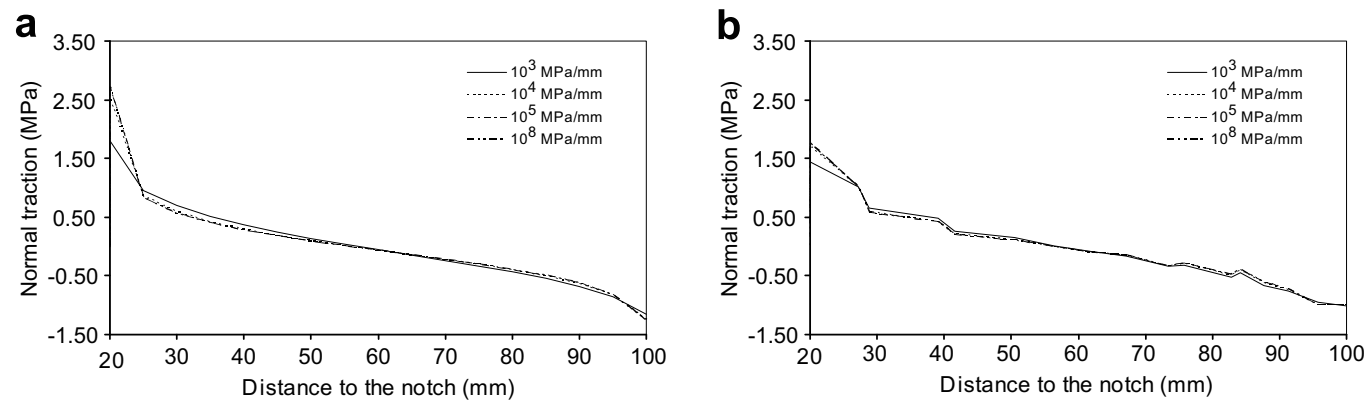

Fig. 8. Traction profile in front of the notch of the beam with the DSDA for: (a) structured mesh and (b) unstructured mesh. 
shear jump along the discontinuity is not automatic. This is why it becomes necessary to enforce the additional linear homogeneous constraint represented in Eq. (24). For this purpose, two methods are adopted in the present work: (i) the Penalty Augmentation method and (ii) the Augmented Lagrange Multipliers method. Note that, although the enforcement of this constraint is crucial in a coarse mesh, it is verified that it lacks importance under mesh refinement.

\section{Material and numerical models}

In [24,25], the strong discontinuity is obtained as a limit case of a weak discontinuity when the crack band width tends to zero. As a consequence, the discrete law can be regarded as a projection of the standard continuum constitutive law into the discontinuity. This continuum strong discontinuity approach (CSDA), although consistent and conceptually appealing, gives rise to a dependence between the bulk material law and the discrete material law, which can lead to some difficulties $[14,48]$.

Conversely, in the DSDA an elastic relationship is considered for the bulk and the discrete material law can be defined independently from the continuum model [35,36]. However, in this case a crack initiation criterion still needs to be given, since no information regarding strain localization is provided from the bulk. In order to derive an initiation criterion, a limit surface is defined in the stress space. This limit surface is defined in the traction space $t_{n}-t_{s}$, where $t_{n}$ is the traction component normal to the discontinuity and $t_{s}$ is the traction component parallel to the discontinuity. The corresponding Mohr representation, in the stress space $\sigma-\tau$, is depicted in Fig. 9. The limit surface is given by:

$$
f=t_{s}^{2}-\frac{f_{t}^{2}+2 c \tan \phi f_{t}-c^{2}}{f_{t}^{2}} t_{n}^{2}-c^{2}\left(1+\tan ^{2} \phi\right)+\left(t_{n}+c \tan \phi\right)^{2}=0
$$

where $\phi$ is the internal friction angle, $c$ is the cohesion and $f_{t}$ is the tensile strength. This surface enables a smooth transition between mode-I fracture and mode-II fracture. Depending on the point where the limit surface is reached, the normal to the new discontinuity $\mathbf{n}_{\Gamma_{d}}$ is defined by the angle $\theta$, such that $\mathbf{n}_{\Gamma_{d}} \cdot \mathbf{e}_{\mathbf{I}}=\cos \theta$. Several examples of plane stress states are represented in Fig. 9: the uniaxial tensile state; uniaxial compression state; an intermediate state and the biaxial compression state. If point $\mathrm{A}$ is reached, the discontinuity initiates according to mode-I fracture, perpendicularly to the direction of $\sigma_{I}(\theta=0)$. From Fig. 9 it can be observed that, unless high values of the compressive normal traction are considered, mixed-mode usually leads to mode-I crack initiation, as experimentally observed. On the other hand, if high values of the compressive normal tractions are attained, the normal to the discontinuity defines an angle $\theta$ with the direction of $\sigma_{I}$, such that:

$$
\theta=\frac{1}{2}\left(\pi / 2-\phi^{\prime}\right),
$$

and mode-II fracture is obtained. In Eq. 50, the angle $\phi^{\prime}$ obeys:

$$
\tan \phi^{\prime}=\left|\frac{\partial t_{s}}{\partial t_{n}}\right| .
$$

Thus, $\phi^{\prime}$ is equal to the internal friction angle $\phi$ in the case of the uniaxial compression test (point B in Fig. 9) and approaches zero under strong biaxial compression (point C in Fig. 9).

Crack closure has to be considered as a realistic feature of the discontinuity model: once the normal jump $w_{n}$ decreases to zero, the crack should not be permitted to close further in order to prevent overlapping of crack faces. This feature is implemented using a penalty function, as done in the discrete-interface approach.

Two energies associated with fracture are defined as material properties: (i) the fracture energy $G_{F}$ and (ii) the energy $G_{F}^{I I}$, defined in mode-II or shear cracking, given by the area below the shear stress-sliding displacement relation obtained in the absence of a normal confining load. The tensile strength $f_{t}$ is a function of an internal scalar variable $\kappa$ according to the exponential flow rule:

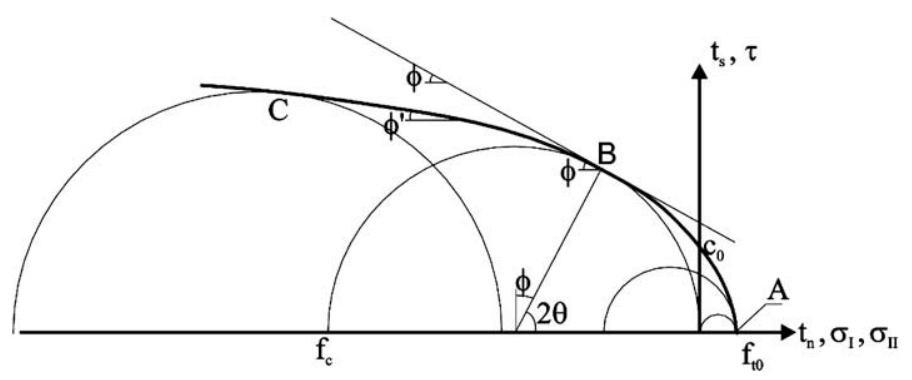

Fig. 9. Limit surface in the traction space. 


$$
f_{t}=f_{t 0} \exp \left(-\frac{f_{t 0}}{G_{F}} \kappa\right)
$$

where $f_{t 0}$ is the initial tensile strength and $\kappa$ is a scalar variable defined below. In [22] several different damage models and a plasticity model are introduced in order to take into account mode-I, mixed-mode and mode-II fracture. Here, both an isotropic and a non-isotropic damage models are presented as examples of both mode-I and mixed-mode fracture, respectively.

\subsection{Isotropic damage law}

In this section the isotropic damage law presented in [22] is described. The constitutive relation is given by:

$$
\mathbf{t}=(1-d) \mathbf{T}_{e l} \mathbf{w},
$$

where $0 \leqslant d \leqslant 1$ is a scalar damage variable and $\mathbf{T}_{e l}$ is the elastic constitutive tensor in which non-diagonal terms are zero and diagonal terms are penalty functions used to prevent overlapping of crack faces under crack closure. The evolution of damage is given by:

$$
d=d(\kappa)=1-\frac{\kappa_{0}}{\kappa} \exp \left(-\frac{f_{t 0}}{G_{F}}\left(\kappa-\kappa_{0}\right)\right)
$$

where $\kappa$ is a scalar variable which takes into account both the maximum positive normal jump component, $\left\langle w_{n}\right\rangle^{+}$, and the maximum shear jump component, $\left|w_{s}\right|$ :

$$
\kappa=\kappa(\mathbf{w})=\max \left\langle w_{n}\right\rangle^{+}+\beta \max \left|w_{s}\right|,
$$

and $\beta$ is discussed below. The internal scalar variable $\kappa$ in Eq. (55) can be considered an equivalent jump ( $\left.\kappa=w_{e q}\right)$ and is a function of the fracture energy $G_{F}$, and of the initial tensile strength $f_{t 0}$, as well as of the displacement jump components. A load function in the displacement jump space is given by:

$$
f=\left\langle w_{n}\right\rangle^{+}+\beta\left|w_{s}\right|-\kappa \leqslant 0 .
$$

The factor $\beta \geqslant 0$ defines the contribution of the shear jump component to the equivalent jump parameter. If $\beta=0$, no contribution from the shear component is considered. In order to define the elastic tensor $\mathbf{T}_{e l}$, an initial value $\kappa_{0} \leqslant \kappa$ is introduced, such that:

$$
\mathbf{T}_{e l}=\frac{f_{t 0}}{\kappa_{0}} \mathbf{I}
$$

where I is the Kronecker tensor. Eq. (53) is the total constitutive equation, where $d$ is a scalar parameter which accounts for the amount of damage through the evolution law given by Eq. (54), such that:

$$
\begin{cases}d=0 & \text { if } \kappa=\kappa_{0} \\ d=1 & \text { if } \kappa=\infty\end{cases}
$$

Replacing Eqs. (57) and (54) into Eq. (53) and writing the constitutive relation in matrix form gives:

$$
\left\{\begin{array}{l}
t_{n} \\
t_{s}
\end{array}\right\}=\frac{\kappa_{0}}{\kappa} \exp \left(-\frac{f_{t 0}}{G_{F}}\left(\kappa-\kappa_{0}\right)\right) \frac{f_{t 0}}{\kappa_{0}}\left[\begin{array}{ll}
1 & 0 \\
0 & 1
\end{array}\right]\left\{\begin{array}{l}
w_{n} \\
w_{s}
\end{array}\right\} .
$$

The crack opening is assumed in mode-I and, at the onset of localisation, $w_{s}=0$ and $t_{s}=0$, whereas $w_{n}=\kappa_{0}$ and $t_{n}=f_{t 0}$.

Differentiating Eq. (59), the incremental constitutive relation can be derived:

$$
\dot{\mathbf{t}}=(1-d) \mathbf{T}^{e l} \dot{\mathbf{w}}-\dot{d} \mathbf{T}^{e l} \mathbf{w}=(1-d) \mathbf{T}^{e l} \dot{\mathbf{w}}-\dot{d} \mathbf{t}^{e l},
$$

where $\mathbf{t}^{e l}$ is the elastic traction vector and:

$$
\dot{d}=\frac{\partial d}{\partial \kappa} \frac{\partial \kappa}{\partial \mathbf{w}} \dot{\mathbf{w}}
$$

Eq. (61), together with Eq. (60), leads to:

$$
\dot{\mathbf{t}}=\left[(1-d) \mathbf{T}^{e l}-\frac{\partial d}{\partial \kappa} \mathbf{t}^{e l} \otimes \frac{\partial \kappa}{\partial \mathbf{w}}\right] \dot{\mathbf{w}}
$$

If $\mathbf{T}^{e l}$ contains the penalty weights used to keep the discontinuity closed before the onset of the localisation, the previous equation can be further developed:

$$
\begin{aligned}
& \frac{\partial d}{\partial \kappa}=\frac{\left(\kappa f_{t 0}+G_{F}\right) \kappa_{0}}{\kappa^{2} G_{F}} \exp \left(-\frac{f_{t 0}}{G_{F}}\left(\kappa-\kappa_{0}\right)\right), \\
& \frac{\partial \kappa}{\partial \mathbf{w}}=\left\{\begin{array}{c}
1 \\
\beta \operatorname{sign}\left(w_{s}\right)
\end{array}\right\},
\end{aligned}
$$


therefore

$$
\frac{\partial d}{\partial \kappa} \mathbf{t}^{e l} \otimes \frac{\partial \kappa}{\partial \mathbf{w}}=\frac{\partial d}{\partial \kappa}\left[\begin{array}{ll}
k_{n} w_{n} & k_{n} w_{n} \beta \operatorname{sign}\left(w_{s}\right) \\
k_{s} w_{s} & k_{s} w_{s} \beta \operatorname{sign}\left(w_{s}\right)
\end{array}\right],
$$

and the following tangential relationship can be written:

$$
\dot{\mathbf{t}}=\left[\begin{array}{cc}
k_{n}\left(1-d-\frac{\partial d}{\partial \kappa} w_{n}\right) & -\frac{\partial d}{\partial \kappa} k_{n} w_{n} \beta \operatorname{sign}\left(w_{s}\right) \\
-\frac{\partial d}{\partial \kappa} k_{s} w_{s} & k_{s}\left(1-d-\frac{\partial d}{\partial \kappa} w_{s} \beta \operatorname{sign}\left(w_{s}\right)\right)
\end{array}\right] \dot{\mathbf{w}} .
$$

If unloading takes place, the rate of damage is zero, $\dot{d}=0$, and both Eqs. (60) and (62) reduce to:

$$
\dot{\mathbf{t}}=(1-d) \mathbf{T}^{e l} \dot{\mathbf{w}} .
$$

Values of $0 \leqslant \beta \leqslant 1$ are adopted herein. A more detailed description of the model can be found in [22].

\subsection{Non-isotropic damage law}

In this section, a 2D version of the model introduced in Wells and Sluys [21] is presented.

A loading function is defined as:

$$
f\left(w_{n}, \kappa\right)=w_{n}-\kappa,
$$

where the internal variable $\kappa$ is taken as the maximum normal relative displacement attained $\left(\kappa=\max w_{n}\right),(\dot{\kappa} \geqslant 0)$. Mode-I fracture initiation is considered. If $f>0$, loading takes place as well as the evolution of damage $(\dot{\kappa}>0)$, whereas if $f<0$, closing of the crack occurs and damage does not grow $(\dot{\kappa}=0)$. An exponential softening law is adopted for the constitutive relation between the normal traction component $t_{n}$ and the normal relative displacement between crack faces (normal jump), given by:

$$
t_{n}=f_{t 0} \exp \left(-\frac{f_{t 0}}{G_{F}} \kappa\right),
$$

where $G_{F}$ is the fracture energy, defined as the amount of energy consumed for the creation of an unit area crack surface and $f_{t 0}$ is the initial tensile strength of the material. The shear traction component $t_{s}$ is related to the sliding relative displacement along crack faces (shear jump $w_{s}$ ) according to:

$$
t_{s}=D_{s 0} \exp \left(h_{s} \kappa\right) w_{s},
$$

where $D_{s 0}$ is the initial shear stiffness at crack initiation, $h_{s}$ is given by:

$$
h_{s}=-\ln \left(D_{s \kappa} / D_{s 0}\right),
$$

and $D_{s \kappa}$ is the shear stiffness which is adopted for an advanced state of damage $(\kappa \gg 0)$. The incremental constitutive relation in matrix form is given by:

$$
\left\{\begin{array}{l}
\dot{t}_{n} \\
\dot{t}_{s}
\end{array}\right\}=\left[\begin{array}{cc}
-\frac{f_{10}^{2}}{G_{F}} \exp \left(-\frac{f_{t 0}}{G_{F}} \kappa\right) & 0 \\
-h_{s} D_{s 0} \exp \left(h_{s} \kappa\right) w_{s} & D_{s 0} \exp \left(h_{s} \kappa\right)
\end{array}\right]\left\{\begin{array}{l}
\dot{w}_{n} \\
\dot{w}_{s}
\end{array}\right\} .
$$

In the case where mode-I crack evolution is considered, the shear stresses are made equal to zero through the enforcement of $D_{s 0}=0$. The secant stiffness matrix is used for unloading, which is given by:

$$
\mathbf{D}_{\text {unloading }}=\left[\begin{array}{cc}
\frac{f_{t 0}}{\kappa} \exp \left(-\frac{f_{t 0}}{G_{F}} \kappa\right) & 0 \\
0 & D_{s 0} \exp \left(h_{s} \kappa\right)
\end{array}\right] .
$$

Crack closure is dealt with in the same manner as described in Section 5.2.

\subsection{Embedded discontinuity technique}

It is assumed that a new embedded discontinuity always crosses the entire parent element; therefore, the crack tip is always located at the element edge. Only straight discontinuities are considered with the direction defined according to the corresponding initiation criterion. For instance, in mode-I fracture, the discontinuity is inserted perpendicularly to the direction of $\sigma_{I}$ which is obtained in the neighbouring area of the crack tip. Although only one discontinuity is allowed in each parent element, more discontinuities can be considered in each element since the additional degrees of freedom will be added to the previous total ones according to Eq. (28), which can be generalized as:

$$
\mathbf{a}^{e}=\hat{\mathbf{a}}^{e}+\sum_{c=1}^{m} \mathbf{H}_{\Gamma_{\mathrm{dc}}}^{e} \tilde{\mathbf{a}}_{c}^{e}=\hat{\mathbf{a}}^{e}+\sum_{c=1}^{m} \mathbf{H}_{\Gamma_{\mathrm{dc}}}^{e} \mathbf{M}_{w c}^{e k} \mathbf{w}_{c}^{e},
$$


where $m$ is the number of discontinuities. Crack path continuity is automatically enforced, according to an algorithm similar to the one presented in [22].

The crack initiation criterion introduced in Section 5 must be verified at the crack tip. Different criteria have been proposed in literature for obtaining the stresses at the tip. In particular, the following are mentioned:

(1) The accurate definition of the stresses near the crack tip is approximated by using special functions taken from linear elastic fracture mechanics $[32,49,50]$;

(2) The mean values of the stress components obtained at the nearest integration points of the crack tip define the stress state adopted for crack evolution [6,51], or

(3) A non-local stress state is adopted near the crack tip, in which case the averaging support is extended beyond the element size [33].

In the examples presented below, the third criterion is adopted, using a Gaussian weight function to smooth out the stresses at the discontinuity tip:

$$
w_{i}=\frac{1}{(2 \pi)^{3 / 2} l^{3}} \exp ^{\left(-\frac{r^{2}}{2 l^{2}}\right)} .
$$

In Eq. (75), $w_{i}$ is the weight for the integration point $i, r$ is the distance between the integration point and the discontinuity tip, and $l$ is a measure of significant distance around the tip. Wells [39] suggests a value of three times the typical element size, whereas Simone et al. [52] evaluates the typical element size in front of the tip. Herein, $l$ is assumed as circa $1 \%$ of the characteristic length, given by:

$$
l_{c h}=\frac{G_{F} E}{f_{t}^{2}} .
$$

Conversely to the former embedded formulations, traction continuity is enforced in a weak manner. As a consequence, the stress field measured in the bulk is not automatically in equilibrium with the traction field measured in the discontinuity, i.e., the envelope surface is not reached simultaneously in the bulk and in the discontinuity. At crack initiation, in order to prevent the traction field at the tip to lie outside the limit surface, a conservative procedure is adopted: the discontinuities are introduced in an earlier stage, in which the stress field in the bulk lies inside the surface.

It is also known that, in the vicinity of the crack tip, stresses in the bulk lying outside the limit stress surface can be found before the crack advances $[6,22,53]$. This can happen both in the former embedded formulations $[10,12-14,22]$ and in the DSDA. In Alfaiate et al. [22] a radius of influence is introduced to prevent secondary cracking near the crack tip. In this paper a similar procedure is adopted such that only the main cracks are allowed to develop.

\section{Numerical examples}

The numerical examples presented in this section consist of a shear band test, a mode-I fracture test and mixed-mode test, and a failure test of a reinforced concrete beam.

\subsection{Shear band test}

First, one finite element $\left(1 \times 1 \times 1 \mathrm{~mm}^{3}\right)$ is subjected to a plane stress state with all nodes fixed except for the loaded node in the horizontal direction (see Fig. 10).

Both bulk and discontinuity constitutive laws are linear elastic; the following parameters are adopted: Young's modulus $E=30 \mathrm{MPa}$; Poisson ratio $v=0.0$; normal stiffness $k_{n}=10^{5} \mathrm{MPa} / \mathrm{mm}$ and shear stiffness $k_{s}=10^{-5} \mathrm{MPa} / \mathrm{mm}$. The small va-
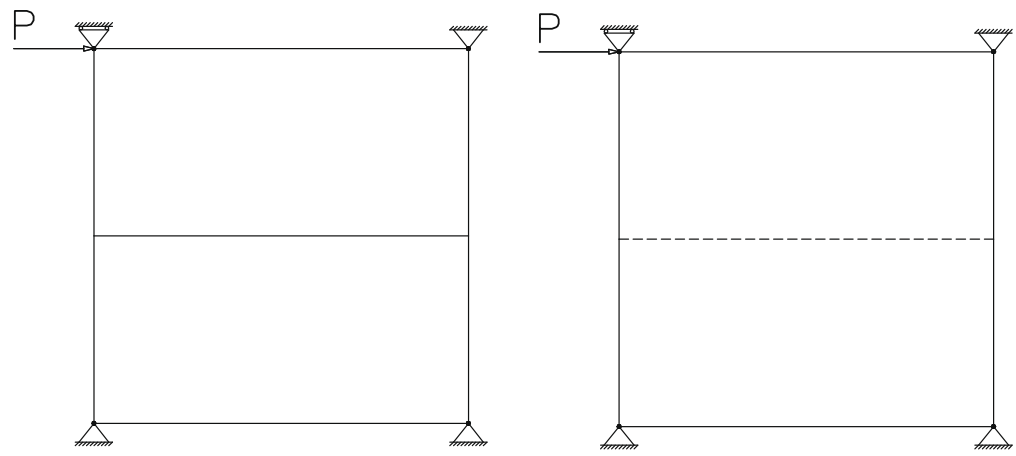

Fig. 10. Mesh used for the numerical tests: (a) discrete-interface and (b) DSDA. 
lue adopted for the Young's modulus emphasizes the bulk deformation, whereas the value $k_{s}=10^{-5} \mathrm{MPa} / \mathrm{mm}$ induces a minimum slip resistance in order to be able to perform the test.

The resulting deformation for both discrete-interface and DSDA is depicted in Fig. $11(P=13.49 \mathrm{~N})$. In Table 1, both the jumps and the top displacement obtained with the DSDA and the discrete-interface approach are shown. The jumps are proportional for both formulations, thus the difference in the overall response is exclusively caused by the bulk.

The result obtained with the DSDA can be interpreted as the superposition of a rigid body motion corresponding to a shear jump of $0.6 \mathrm{~mm}$ (layer 2, in Fig. 12), with the bulk deformation under the assumed boundary conditions (layer 1, in Fig. 12).

This example is illustrative of the behaviour of an element enriched with the DSDA. Adopting a finer mesh, represented in Fig. 13, the deformed mesh presented in Fig. 14 is obtained.

In this case, the differences between the discrete-interface and the DSDA practically vanish. The same would occur if a more realistic, less soft material had been used; for instance, concrete exhibits a Young's modulus 1000 bigger than the one adopted in these tests.

\subsection{Three point bending beam}

A three point bending beam represented in Fig. 15, with a thickness of $50 \mathrm{~mm}$, is analysed assuming a plane stress state. The material parameters are: Young's modulus $E=30 \mathrm{GPa} ; v=0.2 ; f_{t}=3.33 \mathrm{MPa}$; fracture energy $G_{F}=0.11 \mathrm{~N} \mathrm{~mm} / \mathrm{mm}^{2}$ and the normal and shear stiffness $k=k_{n}=k_{s}=10^{9} \mathrm{MPa} / \mathrm{mm}$. The isotropic damage law referred to in Section 5.1 is adopted.

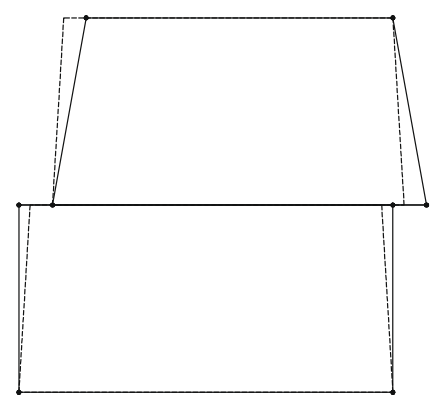

Fig. 11. Deformed mesh (reduced 10 times): discrete-interface (continuous) and DSDA (dashed).

Table 1

Shear jumps and horizontal displacement of the loaded node for discrete-interface and DSDA.

\begin{tabular}{llll}
\hline & Shear jumps $(\mathrm{mm})$ & Horizontal displacement $(\mathrm{mm})$ \\
\cline { 2 - 4 } & Left jump & Right jump & \\
\hline Discrete-interface & 0.9 & 0.9 & 1.8 \\
DSDA & 0.6 & 0.6 & 1.2 \\
\hline
\end{tabular}

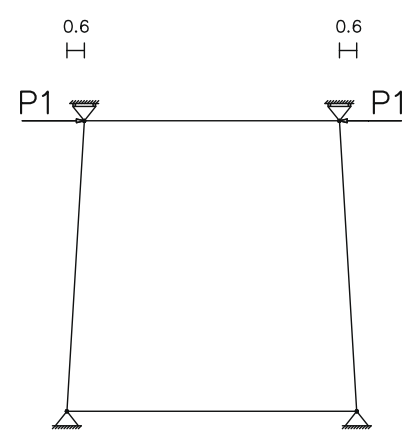

layer 1
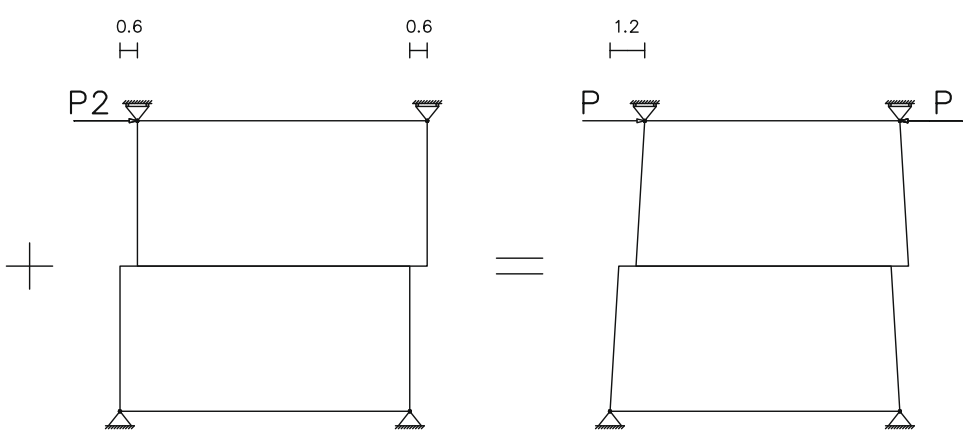

layer 2

Fig. 12. Deformed mesh (reduced 10 times) of DSDA: contribution of each layer to the overall response. 

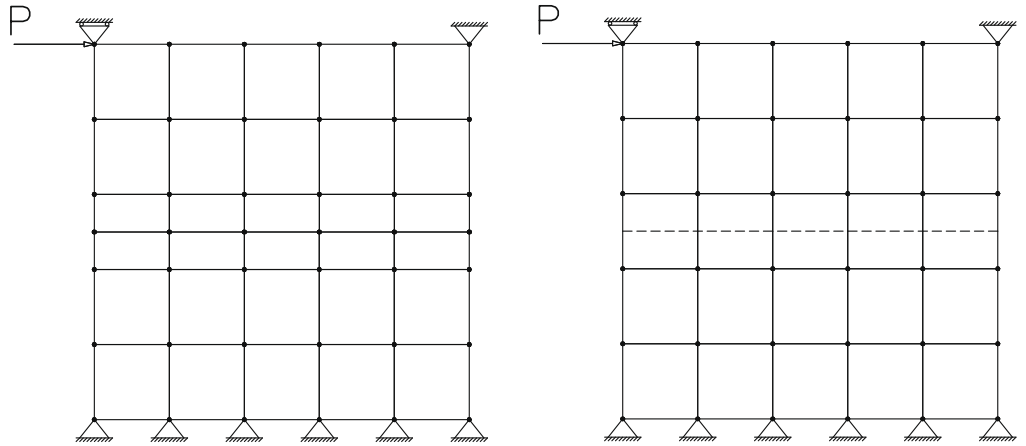

Fig. 13. Coarse mesh used for the numerical tests: (a) discrete-interface and (b) DSDA.

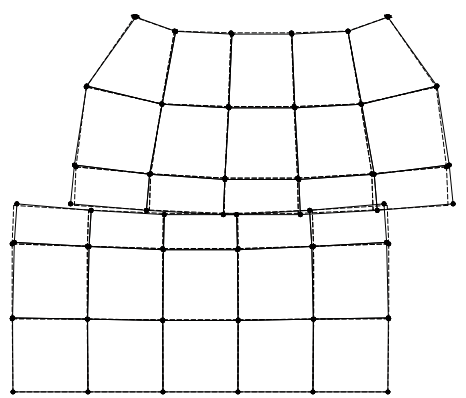

Fig. 14. Deformed coarse mesh (reduced 10 times): discrete-interface (continuous) and DSDA (dashed).

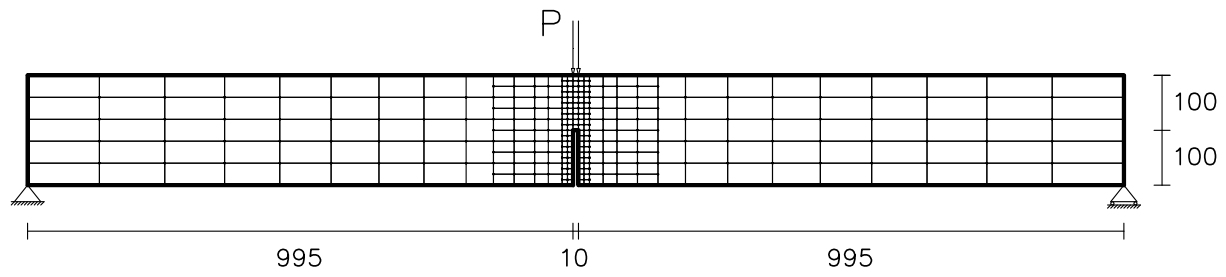

Fig. 15. Three point bending beam ( $\mathrm{mm})$.

In this example the discontinuity is inserted when the maximum principal stress component obtained from the averaged stress tensor at the crack tip reaches $0.4 \mathrm{ft}$.

In Fig. 16 the load-vertical displacement curves obtained are presented for the discrete-interface, the prescribed DSDA and the DSDA. The prescribed DSDA is obtained by predefining the complete discontinuity path for the DSDA since the beginning of the numerical analysis. From the comparison between the results, it can be concluded that all the adopted formulations are similar. Additionally, it becomes clear that it is possible to use the DSDA as an interface like element (see Fig. 17).

\subsection{Single edge notched beam}

A single edge notched beam tested by Schlangen [54] is simulated in this section.

The material parameters are the following: Young's modulus $E=35 \mathrm{GPa} ; v=0.15 ; f_{t}=3.0 \mathrm{MPa}$ and fracture energy $G_{F}=0.1 \mathrm{~N} ; \mathrm{mm} / \mathrm{mm}^{2}$. The normal stiffness adopted is $k_{n}=10^{5} \mathrm{MPa} / \mathrm{mm}$, whereas the shear stiffness is $k_{s}=10^{3} \mathrm{MPa} / \mathrm{mm}$.

Two meshes are initially studied, a coarse and a fine mesh, with 458 and 1604 bilinear finite elements, respectively (see Fig. 18).

The load is controlled using the arc length method in which the monotonic increase of the relative crack mouth sliding displacement (CMSD) of the notch is enforced. The crack path is represented in Fig. 19, while the CMSD vs. load curve is represented in Fig. 20. The deformed meshes are depicted in Figs. 21. 


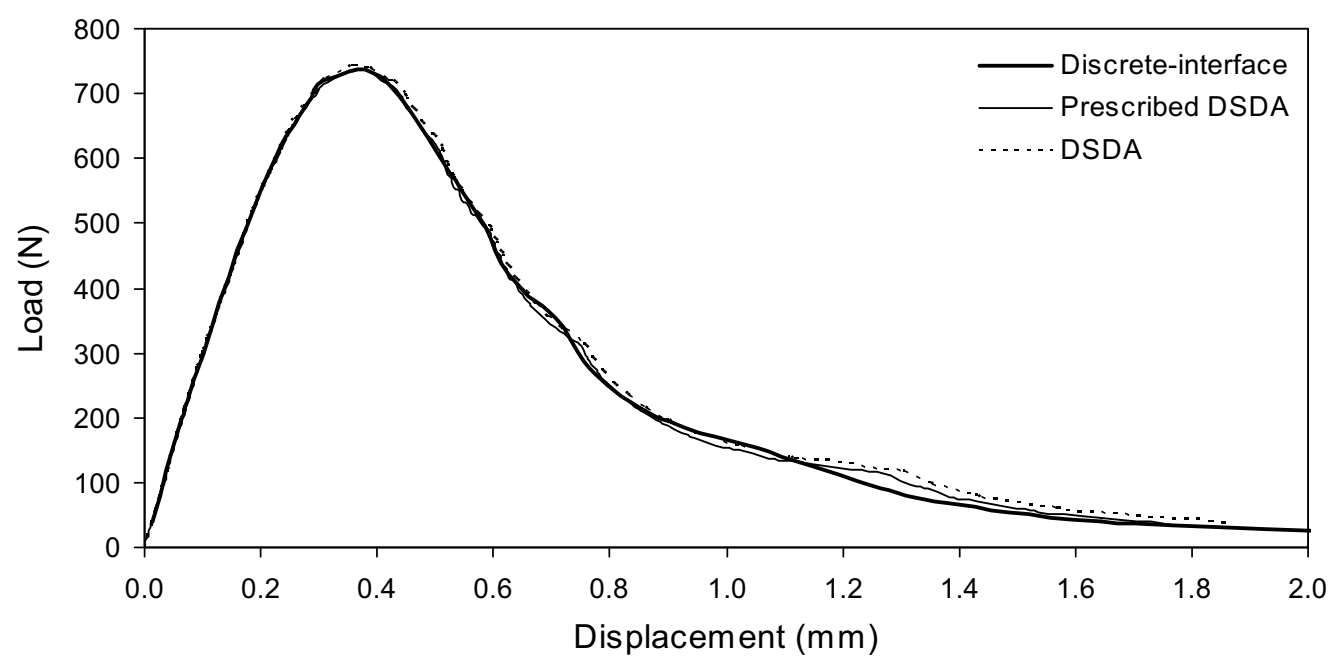

Fig. 16. Three point bending beam: load-vertical displacement curves for the loaded node.

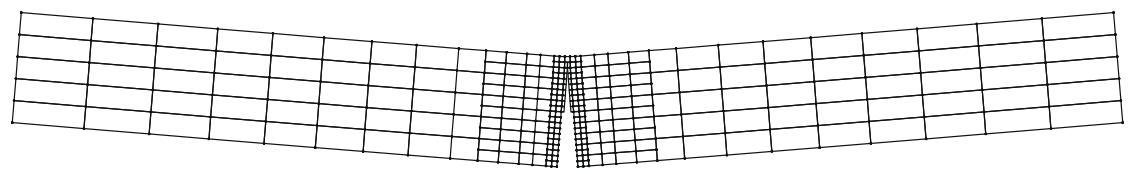

Fig. 17. Three point bending beam: DSDA deformed mesh (magnified 100 times) during softening when the vertical displacement of the loaded node is $u_{y}=0.8 \mathrm{~mm}$.
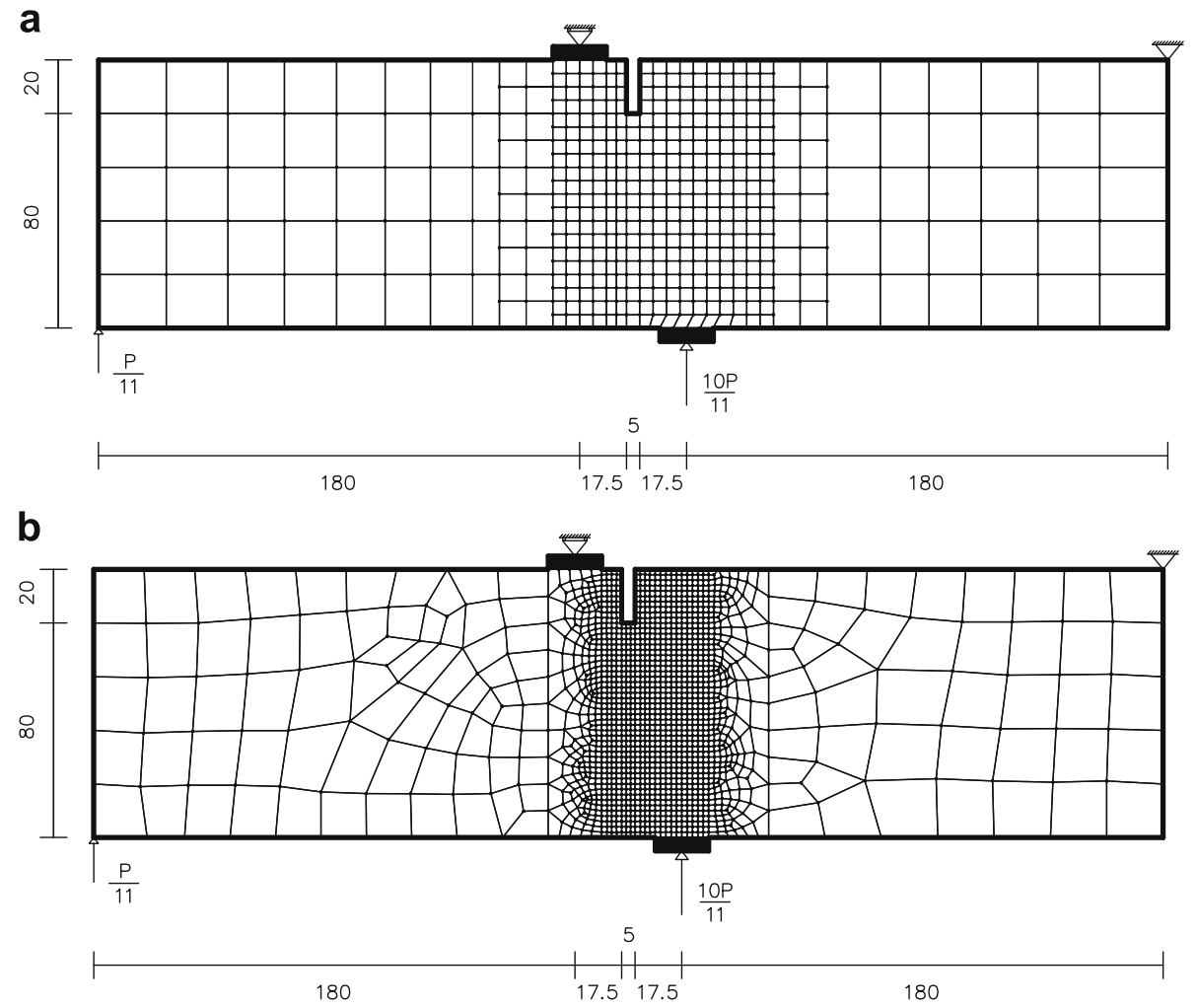

Fig. 18. Single edge notched beam [54]: (a) coarse and (b) fine mesh (mm). 


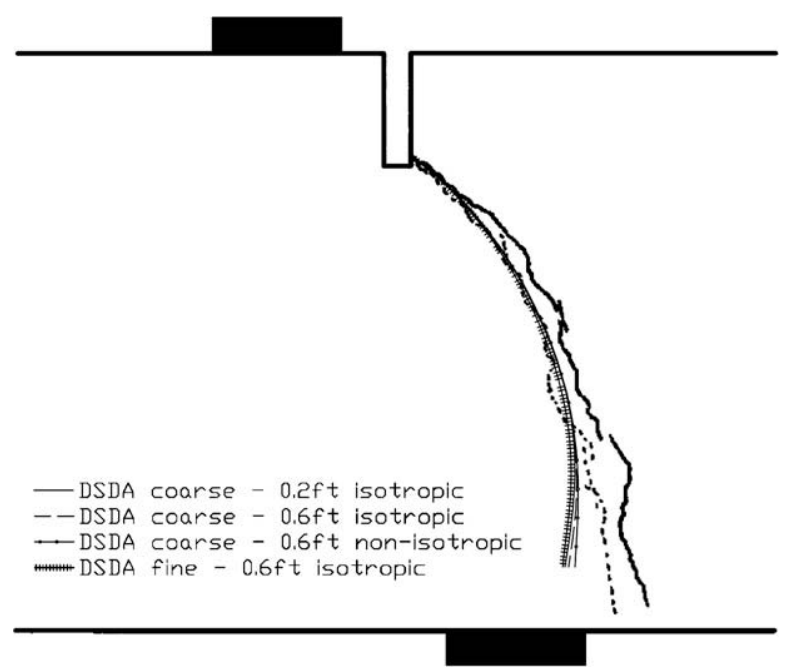

Fig. 19. Single edge notched beam [54]: crack path during softening for $P=20 \mathrm{kN}$, superposed with experimental results.

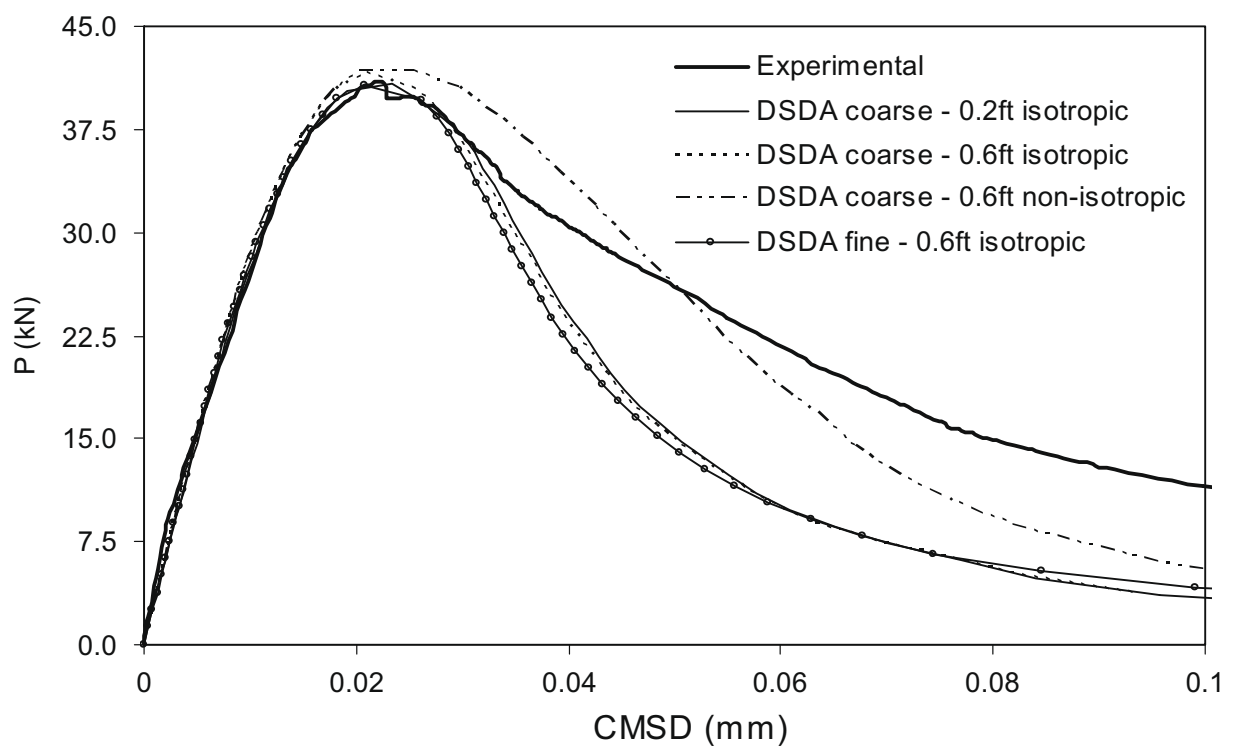

Fig. 20. Single edge notched beam [54]: load-CMSD curves.

When the isotropic constitutive law for mode-I fracture is adopted, experimental and numerical pre-peak results are quite similar. It must be emphasized that both CMSD vs. load curve and crack path for the coarse and fine meshes are identical, leading to the conclusion that good results can be achieved with relatively coarse meshes. Moreover, it can be noticed that the crack path does not change if the discontinuity is inserted at an early stage: as shown in Fig. 20, the same results are obtained if the new discontinuities are inserted when the averaged $\sigma_{I}$ value at the tip changes from $0.2 \mathrm{ft}$ to $0.6 \mathrm{ft}$. This is due to the fact that the principal stress orientation does not significantly change during the monotonic loading.

The numerical crack path is similar to the experimentally obtained crack path until the final portion of the propagation is reached - Fig. 19. From this point on, the numerical path shifts inwards the bottom support, whereas the experimental crack path evolves towards outside the support. Near the support there is a zone of high stress gradients, which is not properly captured by the meshes used. However, this last stage of crack propagation does not seem important for the overall softening response of the specimen.

After the peak load is reached, the numerical results are more brittle than the experimental results. According to [22], this is due to the fact that an isotropic mode-I traction-jump law is used. A better agreement is obtained using a non-isotropic traction-jump, as shown in Fig. 20. 

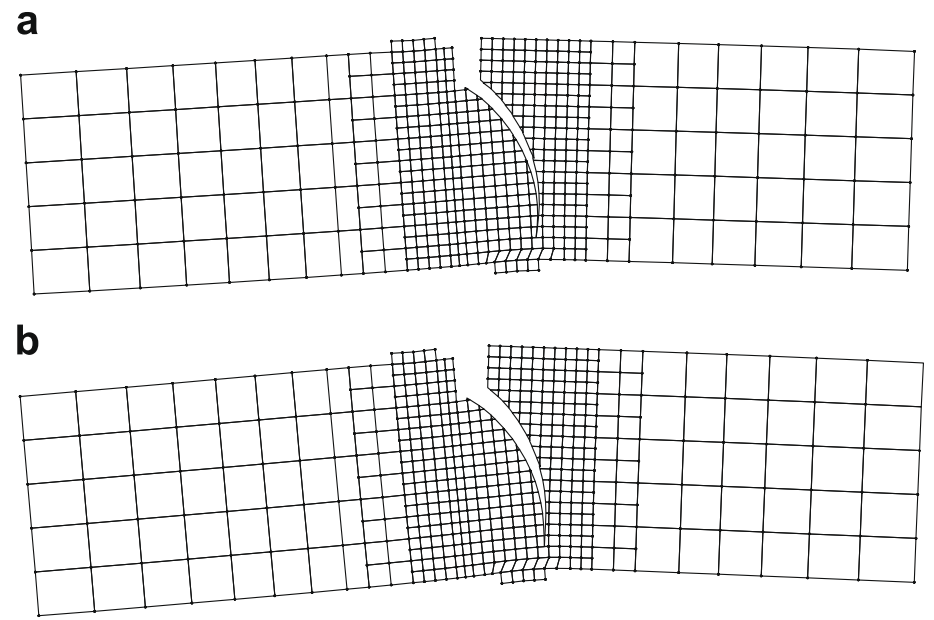

Fig. 21. Single edge notched beam: deformed coarse mesh (magnified 100 times) during softening for $P=20 \mathrm{kN}$ : (a) isotropic and (b) non-isotropic constitutive model.

\subsection{Nooru-mohamed test}

This example consists of a double-edged-notched specimen subjected to mixed-mode fracture, experimentally tested by Nooru-Mohamed [55]. The $200 \times 200 \times 50 \mathrm{~mm}^{3}$ specimen has two $25 \times 5 \mathrm{~mm}^{2}$ horizontal notches located at half height as shown in Fig. 22. Two L-shaped steel frames were glued to the specimen and the loading was applied at the top steel frame. One of the experimental load paths is numerically simulated: first, a horizontal force $P_{h}$ is progressively applied until the value $10 \mathrm{kN}$ is reached. Then, the force is kept constant and a vertical displacement $u_{v}$ is gradually increased (Fig. 22).

The material parameters are taken from [55]: Young's modulus $E=30 \mathrm{GPa} ; v=0.2 ; f_{t}=3.0 \mathrm{MPa}$ and fracture energy $G_{F}=0.11 \mathrm{~N} \mathrm{~mm} / \mathrm{mm}^{2}$. The cohesion $c_{0}$ is estimated using Mohr's rupture theory for brittle materials:

$$
c_{0}=\frac{1}{2} \sqrt{|f c| f t} \approx 5 \mathrm{MPa}
$$

with a compressive strength equal to $38 \mathrm{MPa}$. The constitutive law adopted for the discontinuity is the isotropic damage law presented in Section 5.1, considering a value of $\beta=f_{t} / c_{0}=0.6$.

Two structured meshes are tested (Fig. 23): (a) a coarse mesh, with 435 bilinear finite elements and (b) a fine mesh with 1630 bilinear finite elements.

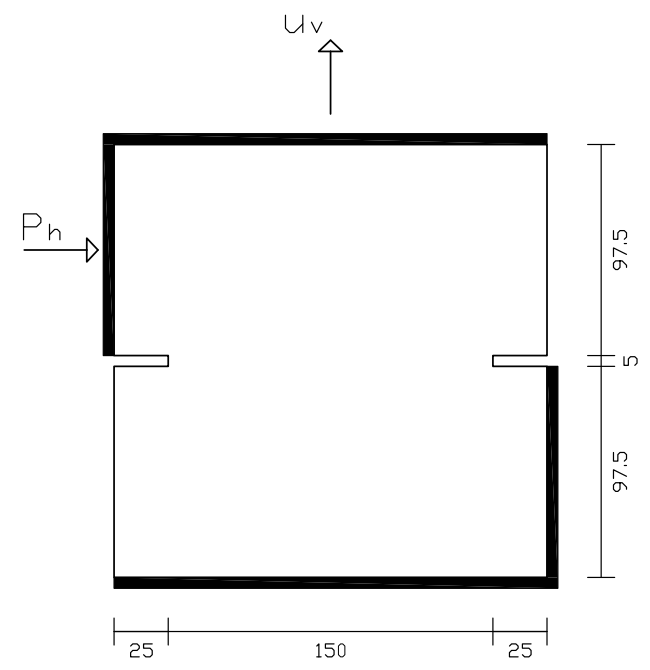

Fig. 22. Mixed-mode fracture test [55]: structural scheme, including loading and boundary conditions (mm). 
The principal stress direction rotates during the increase of the vertical displacement $u_{v}$ causing the crack path to curve. Therefore, the discontinuity must be introduced just before crack opening, when the crack orientation is correctly defined. Two different initiation criteria are applied. When the discontinuity is inserted for an averaged maximum stress component of $0.4 \mathrm{ft}$ at the tip, the numerically obtained crack path is significantly different from the experimental one (Fig. 24a). If the discontinuity is inserted when the averaged maximum stress at the tip reaches $0.8 \mathrm{ft}$, the crack path is similar to the experimentally obtained path as shown in Fig. 24b. In both cases, the crack paths are insensitive to mesh refinement.

The load-vertical displacement curve is depicted in Fig. 25. A small difference between the peak load obtained with the coarse and fine meshes is obtained. Note that the discontinuity always crosses a complete element each time the initiation criterion is reached at the discontinuity tip. As a consequence, a smaller value of the load is needed in the coarse mesh to reach the same tip position (Fig. 26).

The experimental peak load is smaller than the obtained numerical values. Several justifications can be taken from literature, namely incorrect assessment of material parameters as well as spurious bending occurring in the experimental tests and giving rise to different crack paths at the front and rear faces of the specimen [56-58]. This is why some authors tend to focus on the crack path only, disregarding the comparison between numerical and experimental load-displacement curves, namely [59].

\subsection{Numerical modelling of RC beams externally reinforced with steel sheets}

The tests consisted of four point bending beams, with a free span of $1800 \mathrm{~mm}$ and a cross section of $80 \times 200 \mathrm{~mm}$. The internal reinforcements adopted were: $2 \varnothing 8 \mathrm{~mm}$ (tensile reinforcement), $2 \varnothing 6 \mathrm{~mm}$ (compressive reinforcement) and $\varnothing 6 / / 0.08 \mathrm{~m}$ (shear reinforcement). The external reinforcement consisted of a steel plate with a $0.68 \mathrm{~cm}^{2}$ cross section (see Fig. 27).

a

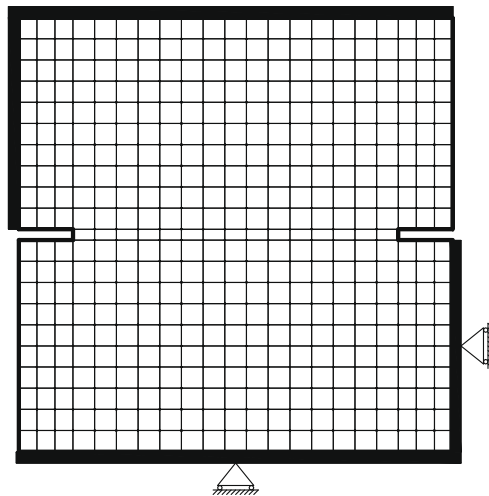

b

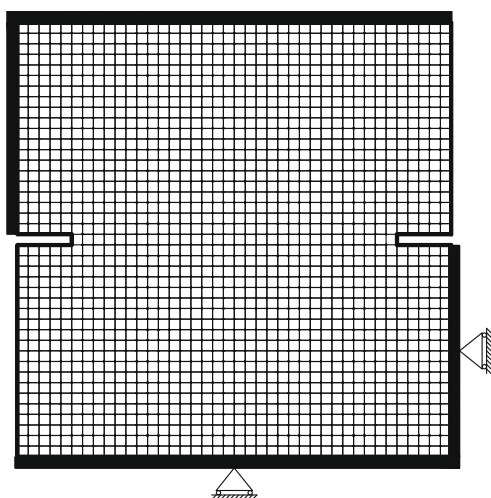

Fig. 23. Mesh used for the numerical tests: (a) coarse and (b) refined mesh.

a

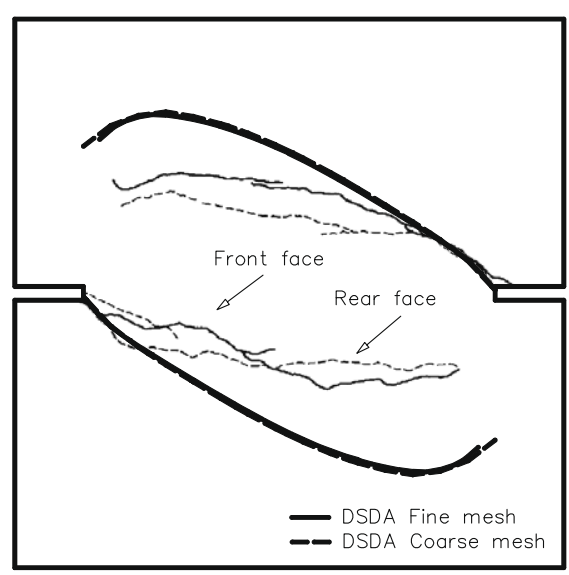

b

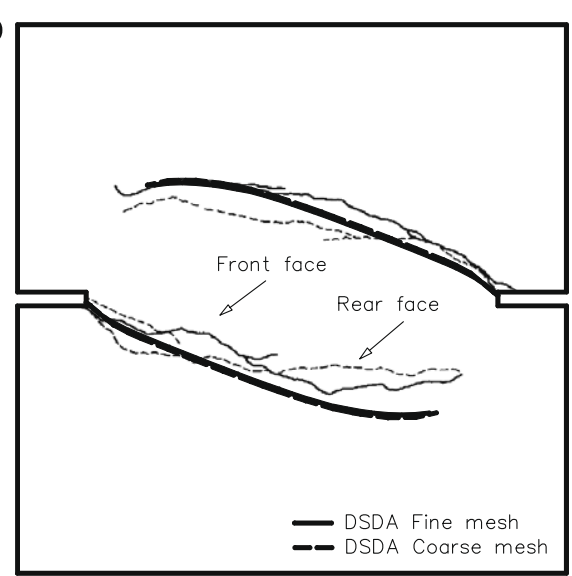

Fig. 24. Crack path, obtained for $u_{v}=0.05 \mathrm{~mm}$, superposed with experimental results taken from [55]: (a) first initiation criterion and (b) second initiation criterion. 


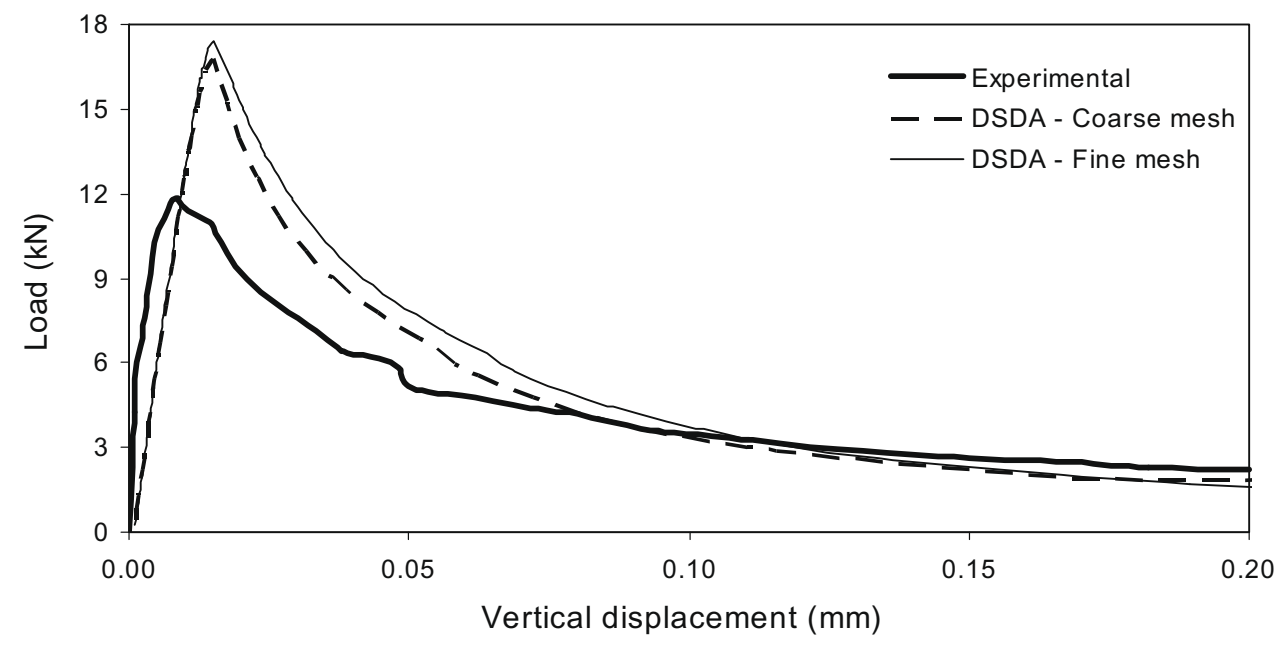

Fig. 25. Load vs. vertical displacement $u_{y}$ superposed with experimental results [55].

a

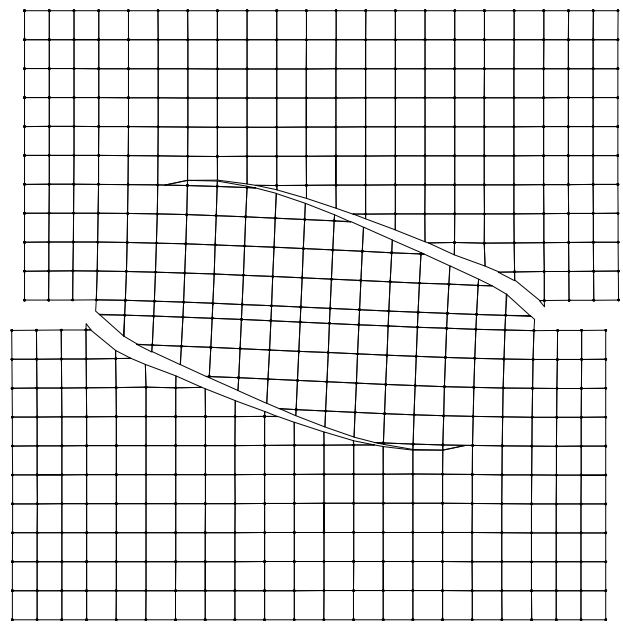

b

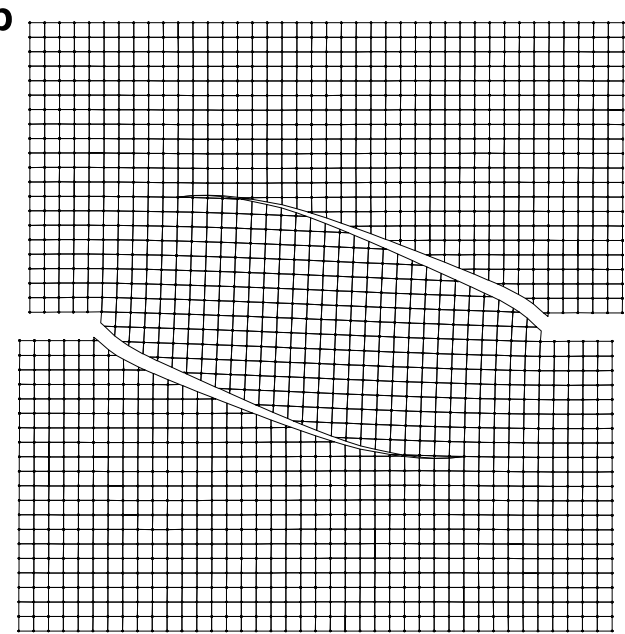

Fig. 26. Deformed mesh (magnified $\times 100$ ), when $u_{v}=0.05 \mathrm{~mm}$ : (a) coarse and (b) fine mesh.

First, a loading was applied and kept constant in order to introduce damage into the structure. Afterwards, repairing of the cracking took place and the external reinforcement was glued with epoxy to the beam. During that time, although creep occurred it is not modelled here, but the corresponding deformation is added to the numerical results in order to allow for a direct comparison with the experimental curves.

The adopted material properties are: concrete grade $\mathrm{C} 20 / 25$, Young's modulus $E_{c}=32 \mathrm{GPa}$, compression strength $f_{c m}=29.67 \mathrm{MPa}, G_{F}=0.056 \mathrm{~N} \mathrm{~mm} / \mathrm{mm}^{2}$ evaluated according to CEB [60]. For the internal reinforcements: Young's modulus $E_{s}=205 \mathrm{GPa}$ and yield stress of $f_{s y}=410 \mathrm{MPa}$. For the external reinforcement: Young's modulus $E_{s R}=200 \mathrm{GPa}$ and yield stress $f_{\text {syR }}=250 \mathrm{MPa}$. It was observed that the failure of the concrete-epoxy-external reinforcement interfaces always occurred at the concrete. Thus, in order to characterize this interface, concrete properties are used in the numerical analysis, namely: cohesion $c_{0}=2.5 \mathrm{MPa}$, shear stiffness $k_{s}=10^{3} \mathrm{MPa} / \mathrm{mm}$ and fracture energy in mode-II, $G_{F}^{I I}=1.38 \mathrm{~N} \mathrm{~mm} / \mathrm{mm}^{2}$. These values are taken from a parametric study presented in [61].

The isotropic damage model is adopted with crack initiation occurring in mode-I. Crushing of concrete is taken into account using perfect plasticity. The internal and external reinforcements are modelled using two-noded truss elements, adopting an elastoplastic constitutive relation. Interface elements are used to model the bond between the concrete and the internal reinforcement, using a constitutive relation adopted from [60]. The bond between external reinforcement, epoxy and concrete is also modelled using interface elements and a mode-II fracture constitutive relation, exhibiting exponential softening.

A sequentially linear approach is used to solve the equilibrium equations, based on a formulation presented in [62]. 


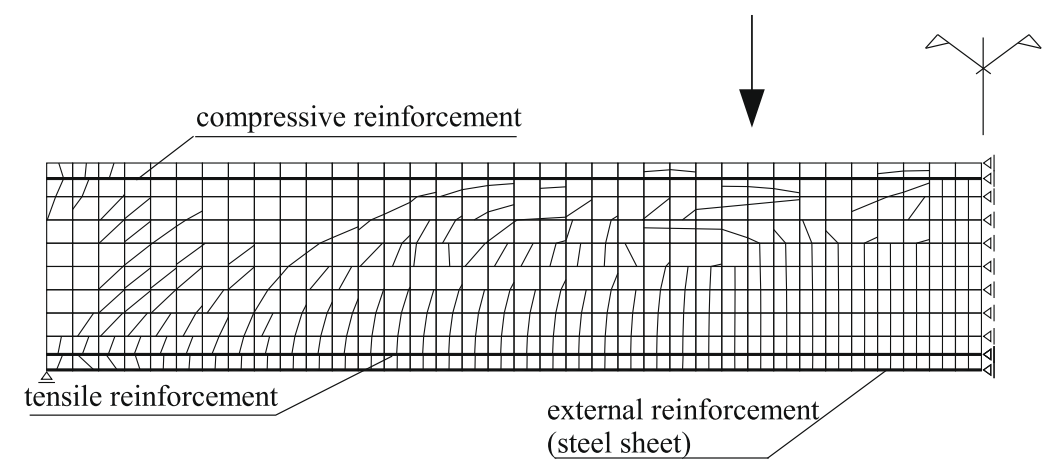

Fig. 27. Schematic representation of the beam, with the micro-cracks numerically obtained immediately after $P_{c r}$ is attained.

The experimental and numerical load-displacement curves obtained at mid-span are presented in Figs. 28 and 29. In the test corresponding to Fig. 28 , the beam was subjected to a smaller initial load, before repairing, than the one applied to the beam in the test corresponding to Fig. 29. In the latter, a more severe degree of damage was initially introduced. Both the cracking load $-P_{c r}$ - and the ultimate load $-P_{u l t}$ - are marked in the graphics. In order to give more information in relation to the $P-\Delta$ graphics shown, some notable points are marked on the curves, namely:

- Point A: the stress $f_{c m}$ (crushing) is first attained;

- Point B: first yielding of internal reinforcement;

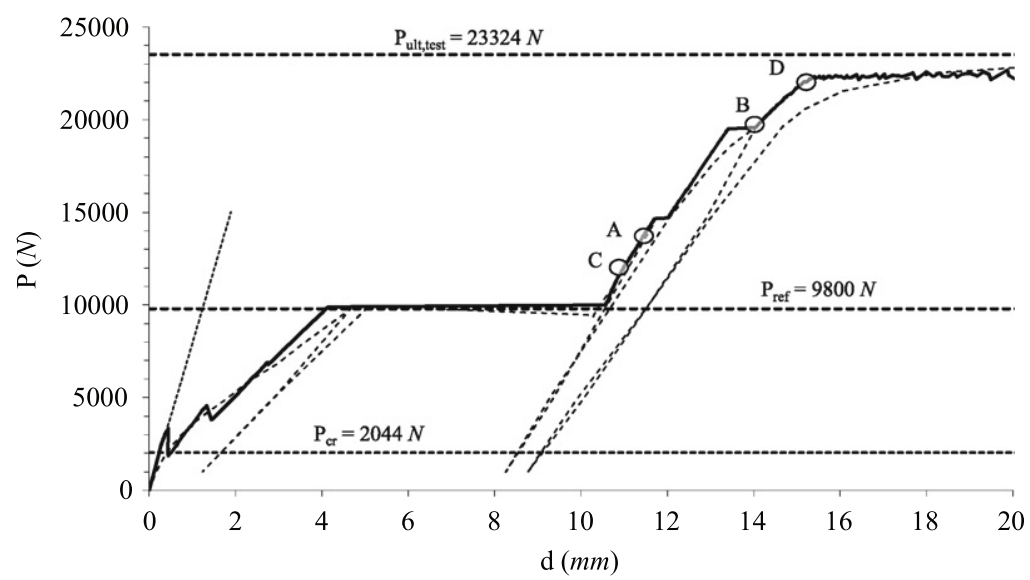

Fig. 28. $P-\Delta$ curves for the beam with low level of initial damage: experimental curve-thin dashed line and numerical curve-thick line.

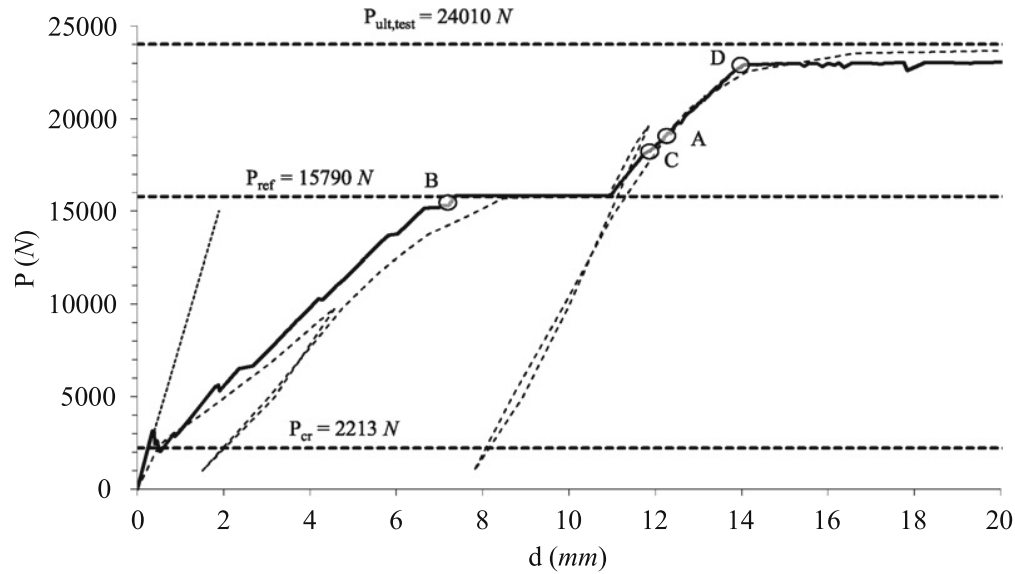

Fig. 29. $P-\Delta$ curves for the beam with high level of initial damage: experimental curve-thin dashed line, numerical curve-thick line. 


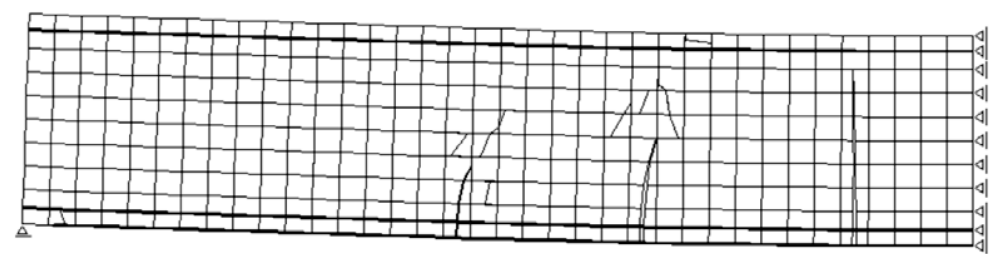

Fig. 30. Crack pattern and deformed mesh at ultimate load (only cracks widths above $10 \mu \mathrm{m}$ are marked).

- Point C: initiation of softening at the epoxy interface;

- Point D: first yielding of external reinforcement.

From Figs. 28 and 29, it is possible to conclude that a good approximation between the numerical and the experimental results is obtained. First, immediately after the load $P_{c r}$ is attained, severe micro-cracks develop as shown in Fig. 27. Afterwards, most micro-cracks tend to close and localization occurs for just a few macro-cracks. After repairing and strengthening, three of these main cracks reopen as shown in Fig. 30, where both the crack pattern and the deformed mesh obtained at the ultimate load are presented. The obtained crack pattern is similar to the one obtained experimentally.

The fact that some micro-cracks tend to close and some others continue to grow, as well as the reopening of some cracks after repairing, leads to convergence problems if a standard Newton-Raphson iterative technique is used. This is why the sequentially linear model is adopted herein, allowing to capture the correct cracking localisation.

\section{Summary and conclusions}

In this paper, a strong embedded discontinuity formulation is introduced, based on a discrete crack approach. This is why the present method is designated as the discrete strong discontinuity approach (DSDA).

In the DSDA, the discontinuity is explicitly inserted into the finite element as if it was an interface element. The parent element is enriched with two or more additional nodes, located along the discontinuity, including the intersection points between the discontinuity and the element edges, with the following characteristics: (i) non-homogeneous jumps are introduced in each parent element; (ii) the edge nodes can be taken as global nodes since they are shared by two elements at a common boundary and (iii) continuous jumps across interelement boundaries are automatically obtained. The adopted variational formulation is the same as the one traditionally used with a discrete-interface approach. The kinematics of the discontinuity consists of a rigid body motion. As a consequence, Simo's orthogonality condition is fulfilled exactly, since the enhanced displacement field induces a null strain field.

The DSDA inherits some characteristics of the discrete-interface approach, namely:

(1) Although the material law adopted for the discontinuity is independent of the material law adopted for the bulk, which is assumed elastic, a criterion needs to be introduced for crack initiation (given in Section 5);

(2) The discontinuities are numerically integrated in the same way as interface elements, giving rise to less spurious effects than the continuum or smeared formulations, in particular if a penalized stiffness matrix is used; this aspect is closely related to the imposition of the rigid body motion, which is dealt with in detail in Section 4.5.

Numerical examples are presented in Section 6, namely a shear band test, mode-I and mixed-mode fracture tests and a failure test of a RC beam externally reinforced with a steel sheet. The following main conclusions can be drawn from these tests:

(1) The jumps obtained with both the DSDA and the discrete-interface approach are similar.

(2) The differences between the discrete-interface approach and the DSDA are due to the bulk deformation which is better represented by the former, since the parent element is split into two different elements; however, for the problems studied and for quasi-brittle materials in general, softening is the phenomenon which influences mostly the global structural behaviour and it is correctly taken into account by the DSDA.

(3) No mesh dependence is found with the DSDA.

(4) The DSDA gives rise to good results using relatively coarse meshes.

(5) In the tests presented, a good approximation of the experimental crack paths and load displacement curves is obtained with the DSDA.

(6) The reinforced concrete tests are well approximated with the DSDA, allowing for the development of the correct mechanisms experimentally observed, namely crushing of concrete, yielding of both the internal and external reinforcements, bond slip of the steel-concrete interfaces and localisation of deformation due to cracking.

As a general conclusion, the DSDA is a powerful tool for the modelling of fracture in quasi-brittle materials. In fact, the use of the DSDA with a sequentially linear approach simulates well cracking of reinforced concrete elements, for which the usual 
iterative techniques often fail to converge. Some other tests have already been successfully simulated, namely failure tests of RC beams strengthened with externally bonded CFRP sheets [63,64].

\section{Appendix A. A numerical integration of the discontinuity stiffness}

The kinematics of the embedded discontinuity in the DSDA is the same as the kinematics of the interface element. Interface elements can be used to model a wide range of problems, where structural interfaces are present. Goodman et al. [65] presented a formulation of a zero-thickness finite element for the numerical simulation of jointed rocks. Goodman simulated failure in tension or shear, rotation of blocks, development of arches and even the collapse pattern for jointed blocks. The applicability of interface elements is not confined to the analysis of rock joints. Further applications can be found in soilstructure interaction [46]. Moreover, modelling of discrete cracking for quasi-brittle materials is also possible [6], as well as the bond between concrete and reinforcement. More recently, those elements have also been used in the study of delamination in layered composite structures [61].

The interface elements can be grouped into classes. According to [45], a first class includes continuous interface elements, whereas a second class is composed by nodal interface elements, which behave like spring elements. An example of the latter are the elements developed by Herrmann [66]. Interfaces can also be simulated with distorted finite elements, where the thickness is reduced. Other numerical procedures include the use of Lagrange multipliers to enforce compatibility between different media, typically in contact problems.

In a plane interface element, the jumps $\mathbf{w}^{e}$ can be obtained by taking the difference between top and bottom displacements, for each pair $i$ of nodes:

$$
d \mathbf{w}^{e}=\mathbf{L}_{w} d \mathbf{a}^{e},
$$

where $\mathbf{L}_{w}$ is a $(2 n \times 4 n)$ matrix, given by:

$$
\mathbf{L}_{w}=\left[\begin{array}{ccccccccc}
1 & 0 & -1 & 0 & \cdots & 0 & 0 & 0 & 0 \\
0 & 1 & 0 & -1 & \cdots & 0 & 0 & 0 & 0 \\
\vdots & \vdots & \vdots & \vdots & \ddots & \vdots & \vdots & \vdots & \vdots \\
0 & 0 & 0 & 0 & \cdots & 1 & 0 & -1 & 0 \\
0 & 0 & 0 & 0 & \cdots & 0 & 1 & 0 & -1
\end{array}\right]
$$

The jump field along the discontinuity is interpolated according to Eq. (26)b, where $\mathbf{N}_{w}^{e}[s(\mathbf{x})]$ contains the interpolation functions, $\mathbf{N}_{w}^{e^{i}}$, for each pair of nodes $i$.

The incremental jump $d \llbracket \mathbf{u} \rrbracket^{e}$ is computed as:

$$
d \llbracket \mathbf{u} \rrbracket^{e}=\mathbf{N}_{w}^{e} d \mathbf{w}^{e}=\underbrace{\mathbf{N}_{w}^{e} \mathbf{L}_{w}}_{\mathbf{B}^{e}} d \mathbf{a}^{e} .
$$

The minimization of the total incremental potential energy gives rise to the stationary requirement:

$$
\mathbf{K}^{e} d \mathbf{a}^{e}=d \mathbf{f}_{e x t}^{e}
$$

with

$$
\mathbf{K}^{\mathbf{e}}=\int_{\Gamma_{\mathbf{d}}} \mathbf{B}^{\mathrm{eT}} \mathbf{T}^{\mathbf{e}} \mathbf{B}^{\mathbf{e}} \mathbf{d} \Gamma_{\mathbf{d}},
$$

where $\mathbf{K}^{e}$ is the tangential stiffness matrix of the interface element and $\mathbf{T}^{e}$ is the discontinuity linearised constitutive relation. Prior to opening, integration of the stiffness matrix and the choice of the penalty weights contained in $\mathbf{T}^{e}$ are of fundamental importance. High initial penalty stiffness are usually introduced to enforce practically zero jumps and must, simultaneously, guarantee that the traction profile is obtained with the necessary accuracy along and through the discontinuity. The choice of the penalty weights is not a closed subject. Basic deficiencies inherent to the element formulation, induced by the adopted numerical integration procedure, can be responsible for spurious oscillations in the traction profile [46].

The interface element is hereafter assumed linear, with $n=2$, in order to compare results with the DSDA. The result of the integration of Eq. (A.5) with the Gaussian quadrature is significantly different from the one obtained with the Newton-Cotes/ Lobatto scheme:

$$
\mathbf{K}_{g}=\frac{A_{d}}{6}\left[\begin{array}{cccc}
2\left[\mathbf{T}^{e}\right] & -2\left[\mathbf{T}^{e}\right] & {\left[\mathbf{T}^{e}\right]} & -\left[\mathbf{T}^{e}\right] \\
-2\left[\mathbf{T}^{e}\right] & 2\left[\mathbf{T}^{e}\right] & -\left[\mathbf{T}^{e}\right] & {\left[\mathbf{T}^{e}\right]} \\
{\left[\mathbf{T}^{e}\right]} & -\left[\mathbf{t}^{e}\right] & 2\left[\mathbf{T}^{e}\right] & -2\left[\mathbf{T}^{e}\right] \\
-\left[\mathbf{T}^{e}\right] & -\left[\mathbf{T}^{e}\right] & -2\left[\mathbf{T}^{e}\right] & 2\left[\mathbf{T}^{e}\right]
\end{array}\right]
$$



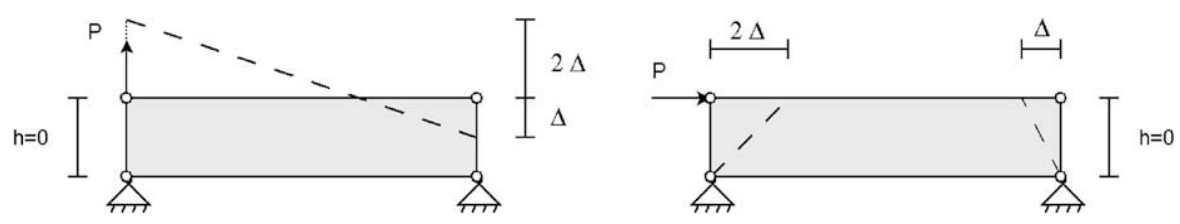

Fig. A.1. Kinematic inconsistency in a linear interface element integrated by Gauss quadrature (interface artificially represented with nonzero thickness).
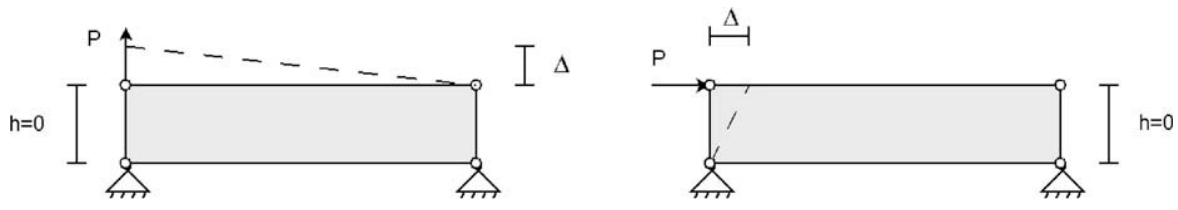

Fig. A.2. Absence of kinematic inconsistency in a linear interface element integrated by trapezoidal rule (interface artificially represented with nonzero thickness).

$$
\mathbf{K}_{t}=\frac{A_{d}}{4}\left[\begin{array}{cccc}
{\left[\mathbf{T}^{e}\right]} & -\left[\mathbf{t}^{e}\right] & 0 & 0 \\
-\left[\mathbf{T}^{e}\right] & {\left[\mathbf{T}^{e}\right]} & 0 & 0 \\
0 & 0 & {\left[\mathbf{T}^{e}\right]} & -\left[\mathbf{T}^{e}\right] \\
0 & 0 & -\left[\mathbf{T}^{\boldsymbol{e}}\right] & {\left[\mathbf{T}^{e}\right]}
\end{array}\right],
$$

where $A_{d}$ is the discontinuity area (thickness $\times$ length) and $\mathbf{K}_{\text {trap }}$ is the Newton-Cotes/Lobatto scheme with two integration points, also known as trapezoidal rule.

In this example, the result obtained using the Gaussian quadrature is coincident with the result obtained for the exact integration. When comparing both $\mathbf{K}_{g}$ and $\mathbf{K}_{t}$, it becomes evident that coupling of the two pairs of nodes is only present in the former. This coupling is pointed by some authors as the cause of tangential traction oscillations (see Figs. A. 1 and A.2). Furthermore, the uncoupled tangential response found with the trapezoidal rule is not necessarily in agreement with reality [46]. However, the necessary shear connectivity between the interface pairs of nodes is provided by the bulk.

Countinho et al. [67] refer to the existence of a spurious kinematical inconsistency in the Goodman interfaces [65] for the Gaussian integration (see Fig. A.1). The oscillations in the displacement field directly induce oscillations in the stress field and the use of high penalty terms increase this effect. The coupling presented between different pair of nodes is also revealed by an eigenvalue analysis of the element stiffness matrix [45,38].

When overintegration is performed with the Newton-Cotes rule, coupling between the different pairs of nodes is also present, similar to the Gaussian quadrature. However, according to [45], the element is less vulnerable to spurious oscillations. Moreover, the oscillation only appears because of the integration point located in the middle of the element. The points located at the extremities are responsible for a better response in relation to the Gaussian quadrature.

Some authors claim that the high gradient of stresses, that usually appears in the neighbourhood of the interfaces, is the main reason for the spurious oscillatory behaviour $[45,68]$. Therefore, it is expected that, upon mesh refinement, the spurious oscillation of the stress profile becomes less important [46]. In fact, Kikuchi and Oden [47] proved, that, in two dimensions, both trapezoidal and Simpson rules give rise to accurate linear and quadratic solutions, respectively, assuming that a sufficiently refined mesh is provided. This is why the trapezoidal Newton-Cotes/Lobatto rule is also adopted in the DSDA, in order to avoid spurious kinematic inconsistencies.

\section{References}

[1] Hillerborg A, Modeer M, Petersson PE. Analysis of crack formation and crack growth in concrete by means of fracture mechanics and finite elements. Cement Concrete Res 1976;6(6):773-82.

[2] Arrea M, Ingraffea A. Mixed mode crack propagation in mortar and concrete. Tech. Rep. 81-13, Dpt. of Structural Engineering, Cornell University, USA; 1982.

[3] Ingraffea A, Saouma V. Numerical modelling of discrete crack propagation in reinforced and plain concrete. In: Sih GC, DiTommaso A, editors. Engineering application of fracture mechanics. Martinus Nijhoff Publishers; 1985.

[4] Bocca P, Carpinteri A, Valente S. Mixed mode fracture of concrete. Int J Solids Struct 1986;27:1139-53.

[5] Alfaiate J, Pires EB, Martins JAC. A finite element model for the study of crack propagation. In: Aliabadi MH, Cartwright DJ, Nisitani H, editors. 2nd international conference on localised damage. Southampton, United Kingdom: Comput Mech Publications and Elsevier Applied Science; 1992. p. 26182.

[6] Alfaiate J, Pires EB, Martins JAC. A finite element analysis of non-prescribed crack propagation in concrete. Comput Struct 1997;63(1):17-26.

[7] Sluys LJ, Berends AH. Discontinuous failure analysis for mode-I and mode-II localization problems. Int J Solids Struct 1998;35(31-32):4257-74.

[8] Dvorkin EN, Cuitino AM, Goia G. Finite elements with displacement interpolated embedded localization lines insensitive to mesh size and distortions. Int J Numer Methods Engng 1990;30:541-64.

[9] Klisinski M, Runesson K, Sture S. Finite element with inner softening band. ASCE J Engng Mech 1991;117(3):575-87. 
[10] Simo JC, Oliver J, Armero F. An analysis of strong discontinuities induced by strain-softening in rate-independent inelastic solids. Comput Mech 1993;12:277-96.

[11] Lotfi HR, Shing PB. Embedded representation of fracture in concrete with mixed finite elements. Int J Numer Methods Engng 1995;38(8):1307-25.

[12] Armero F, Garikipati K. An analysis of strong discontinuities in multiplicative finite strain plasticity and their relation with the numerical simulation of strain localization. Int J Solids Struct 1996;33(20-22):2863-85.

[13] Larsson R, Runesson K. Element-embedded localization band based on regularized displacement discontinuity. ASCE J Engng Mech 1996;122(5):402-11.

[14] Oliver J. Modelling strong discontinuities in solid mechanics via strain softening constitutive equations. Part 1: Fundamentals. Int J Numer Methods Engng 1996;39(21):3575-600.

[15] Oliver J. Modelling strong discontinuities in solid mechanics via strain softening constitutive equations. Part 2: Numerical simulation. Int J Numer Methods Engng 1996;39(21):3601-23.

[16] Ohlsson U, Olofsson T. Mixed-mode fracture and anchor bolts in concrete analysis with inner softening bands. ASCE J Engng Mech 1997;123:1027-33.

[17] Jirásek M, Zimmermann T. Embedded crack model: I. Basic formulation. Int J Numer Methods Engng 2001;50:1269-90.

[18] Jirásek M, Zimmermann T. Embedded crack model. Part II: Combination with smeared cracks. Int J Numer Methods Engng 2001;50:1291-305.

[19] Wells GN, Sluys LJ. Analysis of slip planes in three-dimensional solids. Comput Methods Appl Mech Engng 2000;190(28):3591-606.

[20] Borja RI. A finite element model for strain localization analysis of strongly discontinuous fields based on standard galerkin approximation. Comput Methods Appl Mech Engng 2000;190:1529-49.

[21] Wells GN, Sluys LJ. Three-dimensional embedded discontinuity model for brittle fracture. Int J Solids Struct 2001;38(5):897-913.

[22] Alfaiate J, Wells GN, Sluys LJ. On the use of embedded discontinuity elements with crack path continuity for mode-i and mixed-mode fracture. Engng Fract Mech 2002;69(6):661-86.

[23] Jirásek M. Comparative study on finite elements with embedded discontinuities. Comput Methods Appl Mech Engng 2000;188:307-30.

[24] Oliver J, Huespe AE. A study on finite elements for capturing strong discontinuities. Int J Numer Methods Engng 2003;56:2135-61.

[25] Oliver J, Huespe AE, Samaniego E, Chaves EWV. Continuum approach to the numerical simulation of material failure in concrete. Int J Numer Anal Methods Geomech 2004;28:609-32.

[26] Linder C, Armero F. Finite elements with embedded strong discontinuities for the modeling of failure in solids. Int J Numer Methods Engng 2007;72(12):1391-433.

[27] Sancho J, Planas J, Gálvez J, Cendón D, Fathy A. Three-dimensional simulation of concrete fracture using embedded crack elements without enforcing crack path continuity. In: Owen DRJ, Nate EO, Suárez B, editors. Computational plasticity, fundamentals and applications, COMPLAS VIII. Barcelona, Spain; 2005.

[28] Sancho JM, Planas J, Cendón DA, Reyes E, Gálvez JC. An embedded crack model for finite element analysis of concrete fracture. Engng Fract Mech 2007;74(1-2):75-86.

[29] Melenk JM, Babuška I. The partition of unity finite element method: basic theory and applications. Comput Methods Appl Mech Engng 1996;139(14):289-314.

[30] Duarte CA, Oden JT. H-p clouds - an h-p meshless method. Numer Methods Partial Diff Eqn 1996;12(6):673-705.

[31] Babuška I, Melenk JM. The partition of unity method. Int J Numer Methods Engng 1997;40(4):727-58.

[32] Möes N, Dolbow J, Belytschko T. A finite element method for crack growth without remeshing. Int J Numer Methods Engng 1999;46(1):131-50.

[33] Wells GN, Sluys LJ. A new method for modelling cohesive cracks using finite elements. Int J Numer Methods Engng 2001;50(2):2667-82.

[34] Simone A, Wells GN, Sluys LJ. From continuous to discontinuous failure in a gradient-enhanced continuum damage model. Comput Methods Appl Mech Engng 2003;192(41-42):4581-607.

[35] Alfaiate J, Simone A, Sluys LJ. Non-homogeneous displacement jumps in strong embedded discontinuities. Int J Solids Struct 2003;40(21):5799-817.

[36] Bolzon G. Formulation of a triangular finite element with an embedded interface via isoparametric mapping. Comput Mech 2001;27:463-73.

[37] Washizu K. Variational methods in elasticity and plasticity. 3rd ed. Oxford: Pergamon Press Ltd.; 1982.

[38] Simone A. Partition of unity-based discontinuous elements for interface phenomena: computational issues. Commun Numer Methods Engng 2004;20(6):465-78.

[39] Wells G. Discontinuous modelling of strain localisation and failure, PhD thesis, Delft University of Technology; 2001.

[40] Malvern LE. Introduction to the mechanics of a continuous medium. New Jersey: Prentice-Hall International; 1969.

[41] Simo JC, Rifai MS. A class of mixed assumed strain methods and the method of incompatible modes. Int J Numer Methods Engng 1990;29(8):1595-638.

[42] Martins JAC. Private communication; 2003.

[43] Alfaiate J, Simone A, Sluys LJ. A new approach to strong embedded discontinuities. In: Bicanic N, deBorst R, Mang H, Meschke G, editors. EURO-C 2003, Computational Modelling of Concrete Structures. Austria: St. Johann im Pongau; 2003.

[44] Rots JG. Computational modeling of concrete fracture, PhD thesis, Delft University of Technology; 1988.

[45] Schellekens JCJ, DeBorst R. On the numerical integration of interface elements. Int J Numer Methods Engng 1993;36(1):43-66.

[46] Kaliakin VN, Li J. Insight into deficiencies associated with commonly used zero-thickness interface elements. Comput Geotech 1995;17(2):225-52.

[47] Kikuchi N, Oden JT. Contact problems in elasticity: a study of variational inequalities and finite element methods. SIAM studies in applied mathematics, vol. 8. Philadelphia; 1988.

[48] Alfaiate J. Strong discontinuities embedded in finite elements. Tech. Rep. Relatório ICIST, DTC n 09/00. Lisboa, Portugal: Instituto Superior Técnico; 2000.

[49] Ingraffea A. Shear cracks. In: Elfgren L editor. Fracture mechanics of concrete structures - from theory to applications. Report of the technical Committee 90-FMA fracture mechanics of concrete-applications. London, United Kingdom: Chapman and Hall; 1989. p. 231-33.

[50] Carpinteri A, Valente S, Bocca P. Mixed mode choesive crack propagation. In: Salama K, Ravi-Chandar K, Taplin D, Rao PR, editors. 7th international conference on fracture (ICF-7). New York, USA: Pergamon Press; 1989. p. 2243-57.

[51] Alfaiate J, Pires EB. A discrete crack numerical model. In: Carpinteri A, Aliabadi M, editors. Computational fracture mechanics in concrete techonology. Southampton, United Kingdom: WIT Press/ Comput Mech Publications; 1999. p. 133-62.

[52] Simone A, Remmers JJC, Wells GN. An interface element based on the partition of unity. Tech. Rep. CM2001.007, Technical University of Delft, Delft, The Netherlands; 2000.

[53] Planas J, Elices M, Guinea GV, Gómez FJ, Cendón DA, Arbilla I. Generalizations and specializations of cohesive crack models. Engng Fract Mech 2003;70(14):1759-76.

[54] Schlangen E. Experimental and numerical analysis of fracture processes in concrete, Ph.D. thesis, Delft University of Technology; 1993.

[55] Nooru-Mohamed MB. Mixed-mode fracture of concrete: an experimental approach, Ph.D. thesis, Delft University of Technology; 1992.

[56] Cervera M, Chiumenti M. Smeared crack approach: back to the original track. Int J Numer Anal Methods Geomech 2006;30(12):1173-99.

[57] Pivonka P, Ozbolt J, Lackner R, Mang HA. Comparative studies of 3d-constitutive models for concrete: application to mixed-mode fracture. Int J Numer Methods Engng 2004;60(2):549-70.

[58] Gasser TC, Holzapfel GA. 3d crack propagation in unreinforced concrete: a two-step algorithm for tracking 3d crack paths. Comput Methods Appl Mech Engng 2006;195(37-40):5198-219.

[59] Feist C, Hofstetter G. An embedded strong discontinuity model for cracking of plain concrete. Comput Methods Appl Mech Engng 2006;195(52):7115-38.

[60] CEB/FIP, Model code 90 - design manual; 1990.

[61] Neto P, Alfaiate J, Almeida JR, Pires EB. The influence of mode ii fracture on concrete strengthened with cfrp. Comput Struct 2004;82(17-19):1495502. 
[62] Rots JG. Sequentially linear continuum model for concrete fracture. In: de Borst GPCR, Mazars J, van Mier JGM, editors. 4th international conference on fracture mechanics of concrete structures - FRAMCOS-4, Paris, France; 2001. p. 831-40.

[63] Neto P, Alfaiate J, Vinagre J. Modelling the behaviour of reinforced concrete beams strengthened with FRP. In: Soares CM, Batista A, Bugeda G, Casteleiro M, Goicolea JM, Martins J, et al., editors. ECCM06, III European conference on comput mech solids, structures and coupled problems in engineering. Portugal: Lisboa; 2006.

[64] Alfaiate J, Domingues T. Numerical modelling of concrete beams reinforced with pre-stressed CFRP. In: Alfaiate J, Aliabadi MH, Guagliano M, Susmel L, editors. 6th international conference on fracture and damage mechanics, FDM07, Madeira, Portugal; 2007.

[65] Goodman RE, Taylor RL, Brekke TL. A model for the mechanics of jointed rock. J Soil Mech Foundations Div 1968;99:637-59.

[66] Herrmann LR. Finite element analysis of contact problems. ASCE J Engng Mech Div 1978;104(5):1043-57.

[67] Coutinho ALGA, Martins MAD, Sydenstricker RM, Alves JLD, Landau L. Simple zero thickness kinematically consistent interface elements. Comput Geotech 2003;30(5):347-74.

[68] Day RA, Potts DM. Zero thickness interface elements - numerical stability and application. Int J Numer Anal Methods Geomech 1994;18(10):689-708. 